\title{
RR Lyrae stars: kinematics, orbits and z-distribution ${ }^{\star}$
}

\author{
G. Maintz and K. S. de Boer
}

Sternwarte der Universität Bonn, Auf dem Hügel 71, 53121 Bonn, Germany

e-mail: [gmaintz; deboer]@astro.uni-bonn.de

Received 12 April 2005 / Accepted 8 July 2005

\section{ABSTRACT}

RR Lyrae stars in the Milky Way are good tracers to study the kinematic behaviour and spatial distribution of older stellar populations. A recently established well documented sample of $217 \mathrm{RR}$ Lyr stars with $V<12.5$ mag, for which accurate distances and radial velocities as well as proper motions from the Hipparcos and Tycho- 2 catalogues are available, has been used to reinvestigate these structural parameters. The kinematic parameters allowed to calculate the orbits of the stars. Nearly $1 / 3$ of the stars of our sample have orbits staying near the Milky Way plane. Of the 217 stars, 163 have halo-like orbits fulfilling one of the following criteria: $\Theta<100 \mathrm{~km} \mathrm{~s}^{-1}$, orbit eccentricity $>0.4$, and normalized maximum orbital $z$-distance $>0.45$. Of these stars roughly half have retrograde orbits. The $z$-distance probability distribution of this sample shows scale heights of $1.3 \pm 0.1 \mathrm{kpc}$ for the disk component and $4.6 \pm 0.3 \mathrm{kpc}$ for the halo component. With our orbit statistics method we found a (vertical) spatial distribution which, out to $z=20 \mathrm{kpc}$, is similar to that found with other methods. This distribution is also compatible with the ones found for blue (HBA and sdB) halo stars. The circular velocity $\Theta$, the orbit eccentricity, orbit $z$-extent and $[\mathrm{Fe} / \mathrm{H}]$ are employed to look for possible correlations. If any, it is that the metal poor stars with $[\mathrm{Fe} / \mathrm{H}]<1.0$ have a wide symmetric distribution about $\Theta=0$, thus for this subsample on average a motion independent of disk rotation. We conclude that the Milky Way possesses a halo component of old and metal poor stars with a scale height of $4-5 \mathrm{kpc}$ having random orbits. The presence in our sample of a few metal poor stars (thus part of the halo population) with thin disk-like orbits is statistically not surprising. The midplane density ratio of halo to disk stars is found to be 0.16 , a value very dependent on proper sample statistics.

Key words. astrometry - stars: kinematics - stars: variables: RR Lyr - Galaxy: halo - Galaxy: structure

\section{Introduction}

Studying the spatial distribution of stellar populations in the Milky Way requires well defined tracer objects. Among the large variety of stars, those of horizontal-branch $(\mathrm{HB})$ nature are well suited. HB stars are core-He burners after the red-giant phase whose envelopes have little $\left(<0.1 M_{\odot}\right)$ to perhaps $0.7 M_{\odot}$, leading to a location on the HB from the very blue to red ( $\mathrm{sdB}$, HBB, HBA, RR Lyr, RHB stars). They had an initial mass on the main sequence of $<2.5 M_{\odot}$ which means that some of the RR Lyrs are only about 1 Gyr old and therefore belong to the younger disk population. In particular the blue HB stars and RR Lyrae variables are easy to find. For the RR Lyrae stars (RR Lyrs) the mean absolute magnitude is well calibrated using data from the HIPPARCos (de Boer et al. 1997a; Fernley et al. 1998). The distances of blue HB stars are easily determined utilising their well defined $M_{V}$. For the blue HB stars, models based on the evolutionary nature allow to derive $T_{\text {eff }}$ and $\log g$ from their spectra, thus leading to a fairly accurate $M_{V}$ as well. Red HB stars are less easy to identify, but the HIPPARCOS data allow to isolate nearby ones from the parallax based CMD (Kaempf et al. 1995).

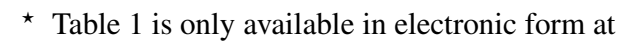
http://www.edpsciences.org
As a result of many years of work on the nature and distribution of sdB stars (Heber 1986; Moehler et al. 1990; Theissen et al. 1993; Saffer et al. 1994; Villeneuve et al. 1995) the idea emerged to include radial velocity and proper motion data allowing to calculate the kinematical properties of these stars, including the calculation of orbits (following Odenkirchen \& Brosche 1992). Several such studies have been carried out thus far.

Using sdB stars, de Boer et al. (1997b) derived from the orbit statistics of 41 stars a $z$-distribution with a scale height of $\simeq 1 \mathrm{kpc}$ plus a hint of the existence of a more extended population. The existence of this latter population was substantiated by Altmann et al. (2004) in a sample of 114 stars, showing a halo group with a $z$-distribution which one can characterise by a scale height of $\simeq 7 \mathrm{kpc}$. Recently, using the CMD identification of red HB stars, Kaempf et al. (2005) presented a similar study of the RHB star distribution. They find a disk-like distribution with a $z$-scale height of perhaps up to $0.8 \mathrm{kpc}$ and an indication for an extended population with a scale height of $\simeq 5 \mathrm{kpc}$.

It is therefore natural to carry out a similar study for RR Lyrae stars since their nature is intermediate to RHB and sdB stars. This has become reliably possible using the recent magnitude limited $(V<12.5 \mathrm{mag})$ complete catalogue of RR Lyrae stars (Maintz 2005), a catalogue free of all kinds of misidentification. 

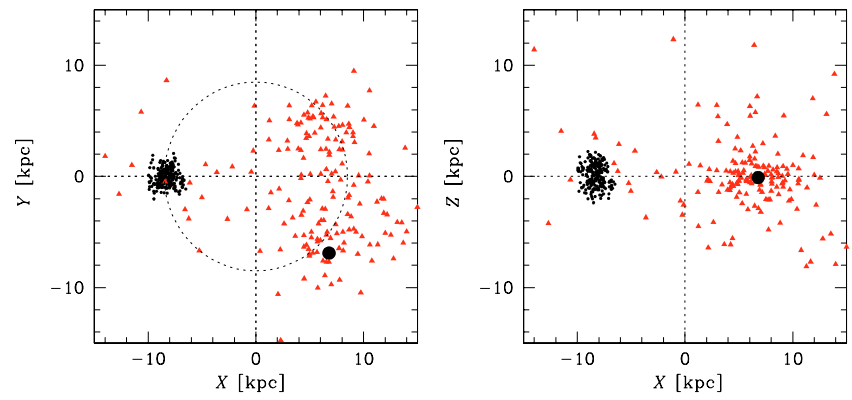

Fig. 1. Distribution of the RR Lyrs of our sample today (hexagons) and $100 \mathrm{Myr}$ ago (triangles), equivalent to about half a galactic revolution. The full circle shows the position of the Sun $100 \mathrm{Myr}$ ago. At left the distribution projected on the Galactic plane, at right perpendicular to the plane (along the $X$-axis). The circle in the left panel shows the $8.5 \mathrm{kpc}$ radius.

Section 2 describes the basic data and in Sect. 3 we present the results of our calculations. We analyse the velocities in Sect. 4 as well as correlations between velocity and other parameters like metallicity. The implications of our findings are discussed in Sect. 5 and a comparison with results from other studies in Sect. 6.

\section{Data}

\subsection{The sample}

The RR Lyrs used for the present investigation are taken from the well documented sample established by Maintz (2005). She checked the identification of RR Lyrs of the GSC with $V<$ 12.5 mag against finding charts and with new observations.

For the present investigation we restricted ourselves to those RRLyrs from her list which were observed in the Hipparcos mission (see The Hipparcos and Tycho Catalogues, ESA 1997; Hip and the Tycho 2 catalogue, Høg et al. 2000 Tyc2). Our sample thus has the best positions and proper motions available. Furthermore, radial velocities have to be known. Of the 104 RR Lyrs found in the Tyc2 we used 62, the other 42 having no reliable $v_{\text {rad. }}$. From the Hip we could use 154 of the 182 RR Lyrs for the same reason. Our data for radial velocity as well as distance from the galactic center came from sources in the literature (Beers et al. 2000; Fernley et al. 1998; Layden 1994). We note that these authors used slightly different methods to come to stellar distances. We thus have investigated 217 RR Lyrs for their kinematic behaviour. Our sample has no kinematical bias. The limits are the limiting magnitude of the Hip and Tyc2 catalogues, combined with the availability of the $v_{\text {rad }}$ data.

The present location of the RR Lyrs of our sample in the $X, Y$ and in the $X, Z$ plane is shown in Fig. 1 with hexagons. Evidently, RR Lyrs have been searched for and found foremost at higher galactic latitudes, avoiding the crowded region of the disk. Furthermore, since our sample is magnitude limited, those stars included while suffering from interstellar extinction have a smaller distance for their apparent magnitude.

The RR Lyrs being located now in the solar neighbourhood spread with time to nearly any galactic location (see Fig. 1,

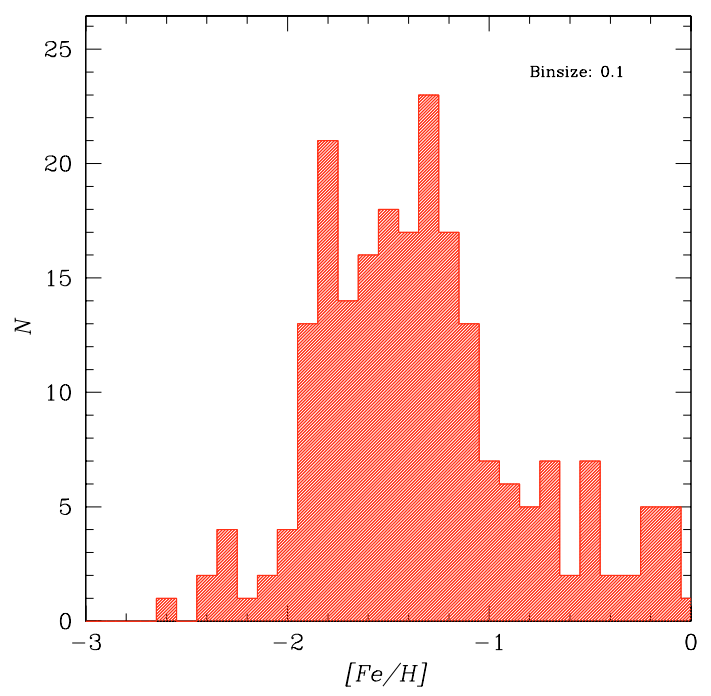

Fig. 2. Histogram of the distribution of our stars in $[\mathrm{Fe} / \mathrm{H}]$. RR Lyrs cover a range from solar like values to very metal poor. Most stars show metallicity $-2<[\mathrm{Fe} / \mathrm{H}]<-1$. Note that several stars have metallicities near the solar value.

triangles). Many must have been at almost any location, both at very small and very large galactocentric radius and at small and large $z$-distance. This means, that our sample of 217 RR Lyrs contains stars one can label as part of a halo, of a thick disk, and of a thin disk population (not claiming these are sharp population distinctions). The last category has kinematics keeping them confined to the galactic plane and thus they may be underrepresented in our sample. Among the 217 are, however, several RR Lyrs with solar abundances (see Sects. 2.2 and 3.2), suggesting that the lack of stars with thin disk kinematics need not be excessive.

\subsection{Metallicity of the RR Lyrs}

The metallicity of our stars is taken from the literature. We use data given by Beers et al. (2000), Fernley et al. (1998) and Layden (1994). As shown in Fig. 2, the metallicity of RR Lyrs ranges from solar values to very low values of -2.6 dex. A broad peak in Fig. 2 shows that most of the stars of our sample have metallicities of $-2<[\mathrm{Fe} / \mathrm{H}]<-1$. A small tail represents stars with still lower metallicity. These abundances indicate, following the usual arguments, that most of the stars are of population II. But there are RR Lyrs with higher metallicity as well: 46 stars have $[\mathrm{Fe} / \mathrm{H}]>-1$. We have in our sample 27 RR Lyrs with a metallicity of $[\mathrm{Fe} / \mathrm{H}]>-0.7$ and 11 stars even have $[\mathrm{Fe} / \mathrm{H}]>-0.3$. This suggests that the lack of relatively young RR Lyrs (likely having kinematics keeping them near the plane) cannot be excessive.

\section{Kinematics and orbits}

We have analysed the distribution of various kinematical aspects of our sample. To get the information for the overall kinematics of the RR Lyrs and to attempt to come to a separation of disk and halo we calculated their orbits. 


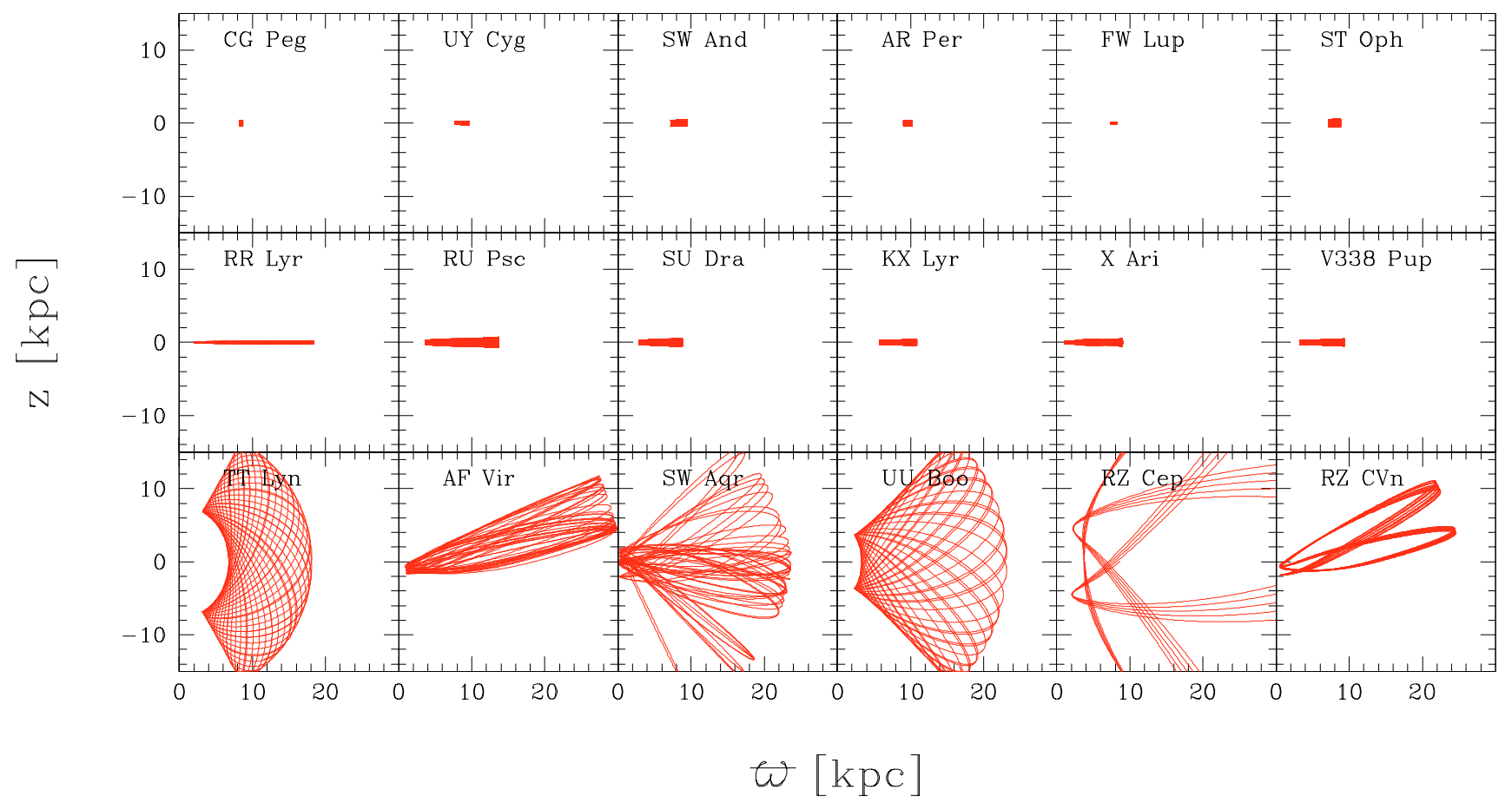

Fig. 3. Meridional plots of some of the calculated RR Lyrae stars orbits. The orbits are calculated over a times pan of 10 Gyr. The top row shows orbits of thin disk star candidates. The middle row shows the orbit of RR Lyrae itself (on the left) and five more stars with elliptic orbits in/near the plane. The bottom row shows orbits of stars moving far out into the halo. For the orbit of the Sun see de Boer et al. (1997b, their Fig. 1).

Table 1. Electronic table; positions, velocities (given in the Galactic euclidic system $X Y Z U V W$ and $\Theta, \Phi)$ and orbital data $\left(R_{\mathrm{a}}, R_{\mathrm{p}}, z_{\max }\right.$ normalised z-extent, nze and eccentricy ecc) of the stars of our sample. This table is only available in electronic form at http://www.edpsciences.org.

\begin{tabular}{cccccccccccccc}
\hline \hline Name & $X$ & $Y$ & $Z$ & $U$ & $V$ & $W$ & $\Phi$ & $\Theta$ & $R_{\mathrm{a}}$ & $R_{\mathrm{p}}$ & $z_{\max }$ & $n z e$ & $e c c$ \\
& {$[\mathrm{kpc}]$} & & {$\left[\mathrm{km} \mathrm{s}^{-1}\right]$} & & & {$[\mathrm{kpc}]$} & & & - & - \\
\hline
\end{tabular}

\subsection{Calculating velocities and orbits}

The observational parameters $\alpha, \delta, d, \mu_{\alpha}, \mu_{\delta}, v_{\text {rad }} \quad$ were transformed into $X, Y, Z, U, V, W$ (for details see de Boer et al. 1997b). Also the orbital velocity projected to the galactic plane, $\Theta$, and the velocity towards the galactic centre, $\Phi$, were calculated. Orbits were calculated over a time span of $10 \mathrm{Gyr}^{1}$ taking steps of 1 Myr. Following de Boer et al. (1997b) the eccentricity, ecc, of the orbit and the apo- and perigalactic distances, $R_{\mathrm{a}}$ and $R_{\mathrm{p}}$, were calculated as well as the maximum $z$-distance reached, $z_{\max }$, and its galactic radial distance normalised $z$-extent, nze,

$e c c=\frac{R_{\mathrm{a}}-R_{\mathrm{p}}}{R_{\mathrm{a}}+R_{\mathrm{p}}}$ and $n z e=\frac{z_{\max }}{\varpi\left(z_{\max }\right)}$.

For the values for our stars see Table 1 available in electronic form.

\footnotetext{
1 This long time allows to show more clearly the shape of the orbits.
}

\subsection{The orbits and the orbit parameters}

The meridional sections of the orbits of RR Lyrs (see Fig. 3) show very different shapes. About half of the stars of our sample have perigalactic distances $R_{\mathrm{p}} \leq 3 \mathrm{kpc}$. $65 \mathrm{RR}$ Lyrs reach a perigalactic distance $R_{\mathrm{p}} \leq 1 \mathrm{kpc}$. Three stars (DR And, AM Vir and AV Vir) reach perigalactic distances less than $0.1 \mathrm{kpc}$. The other extreme is represented by 5 RR Lyrs (CI And, AL CMi, TW Lyn, AR Per and SS Psc) which have perigalactic distances more than $9 \mathrm{kpc}$ and always stay beyond the solar circle.

Of the 217 RR Lyrs 63 have boxy orbits and stay close to the plane. A subset of 29 makes only small excursions in $\varpi$ (Fig. 3 top row), 34 have planar but very eccentric orbits (Fig. 3 middle row). The orbit of RR Lyr itself is not like that of a disk population star (even although its $z_{\max }$ is only $0.21 \mathrm{kpc}$ and its $n z e=0.01$ ), because its orbit has $e c c=0.8$ with $R_{\mathrm{a}}=18.4 \mathrm{kpc}$ and $R_{\mathrm{p}}=2.1 \mathrm{kpc}$.

154 of the RR Lyrs show orbits resembling those of the bottom row. The shape of these orbits is really chaotic, and shows movements perpendicular to the plane. These stars partly have orbits going to very small galactocentric distances and some of them reach very large apogalactic distances, with as an extreme $R_{\mathrm{p}} \simeq 51 \mathrm{kpc}$ (for $\mathrm{SX}$ Aqr).

The statistics of the circular component $\Theta$ of the velocity is shown in Fig. 4. In our sample we have 87 RR Lyrs with retrograde orbits. We compared the characteristics of these stars with the prograde ones. Among the retrograde part of our sample there are no stars with a metallicity $[\mathrm{Fe} / \mathrm{H}]>-0.9$ (see Sect. 4 upper left panel). Considering the retrograde group separately shows that the peak at high eccentricities in Fig. 5 (left panel) is nearly completely due to the retrograde RR Lyrs, 


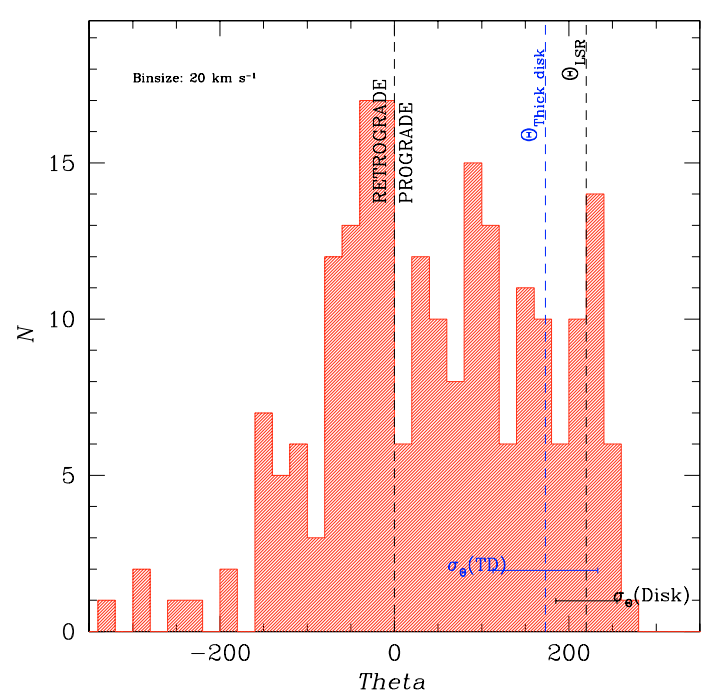

Fig. 4. Histogram of the distribution of our stars in $\Theta\left(\Theta_{\odot}=\right.$ $220 \mathrm{~km} \mathrm{~s}^{-1}$ ). Some stars show circular velocities regarded to be characteristic for the thick and thin disk, respectively (see dashed lines). $\Theta_{\text {LSR }}, \Theta_{\text {thick disk }}$ and the velocity dispersions for thick and thin disk as found by Ojha et al. (1994) are indicated. Most RR Lyrs are halo stars and the high peak represents the RR Lyrs with retrograde motion.

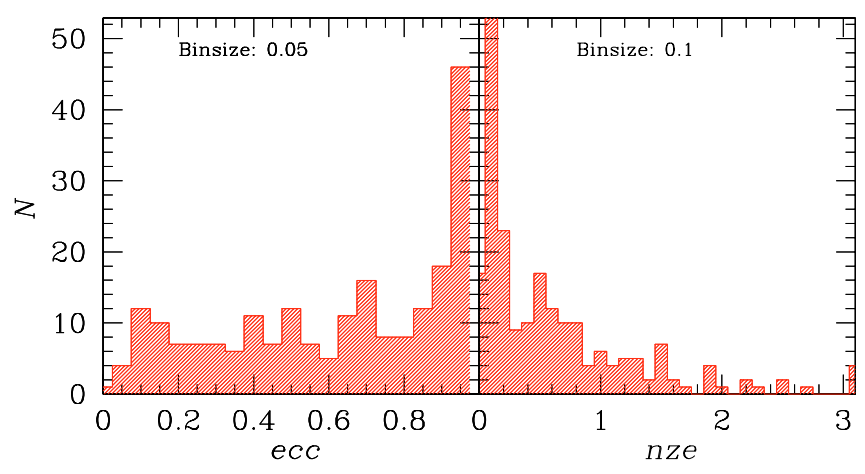

Fig. 5. Histograms showing the distributions of ecc (at left) and nze (at right) for the RR Lyrae stars of the sample. Note the flat distribution of ecc at low values and the peak at $e c c \simeq 0.9$. Values of $n z e$ lie predominantly between 0 and 1 , with a local minimum at $n z e \simeq 0.4$ and a long tail up to $n z e=3$.

while the stars with prograde orbits show a flat distribution over the hole metallicity range.

The eccentricities ecc of the orbits as well as the values of nze span a large range (see Fig. 5).

The eccentricity of the orbit of the majority of our stars is ecc $>0.45$. The distribution has an absolute maximum at $e c c=0.9$ and a shallow local minimum at $e c c \simeq 0.6$.

The distribution of the normalised $z$-extents shows a maximum at low values nze $<0.2$, a local minimum at $n z e=0.4$ followed by another peak at $n z e \simeq 0.6$. There are 109 RR Lyrs with nze $\leq 0.4$ and only $45 \mathrm{RR}$ Lyrs reach nze $\geq 1$. A separation in stars with prograde and retrograde orbits does not change the appearance of the nze diagram. Both groups show high peaks at low values of nze and a long flat tail.

\subsection{Kinematic parameters and population type}

The shape of a stellar orbit gives an indication of the population a star belongs to. Disk-population stars have orbits similar to those of the younger galactic stars, being rather circular and staying close to the plane. These are the ones most likely having been born in the disk. It is, from our data, not easy to discriminate between so-called thin- and thick-disk stars, a distinction which is not well defined anyway. The halo population consists of stars whose orbits are not akin to disk-like galactic rotation. They most likely have been born outside the disk of the Milky Way.

Using the above criteria (which were largely set by the distribution of the parameters of our stars as discussed above using Figs. 4 and 5), one can now roughly sort the stars according to populations. We define halo stars as those having $\Theta<100 \mathrm{~km} \mathrm{~s}^{-1}$ or ecc $>0.4$ or $n z e>0.4$. Each one of the criteria alone suffices to make the star a non-disk born star, and thus a star belonging to the halo population.

Our statistics shows that out of the total of 217 stars, 140 stars have $\Theta<100 \mathrm{~km} \mathrm{~s}^{-1}, 153$ stars have $e c c>0.4$, and 108 stars have $n z e>0.45$, while 163 stars fulfil at least one of the criteria. Or, there are (kinematically) just 54 classic disk stars in our sample.

\section{4. z-probability plot and scale height}

The sum of the orbits was used to derive the associated probability distribution of the $z$-distances of our RR Lyr star sample. This distribution is equivalent to the statistical $z$-density gradient of the sample (for further details see de Boer et al. 1997b). One may fit the distribution with an exponential

$\ln N(z)=\ln N_{0}-\frac{z}{h}$

with $N_{0}$ being the density in the local galactic plane and $h$ the scale height. The slope of the $\log N$ vs. $z$ distribution gives the scale height. Figure 6 shows our $z$-distance statistics. It turns out the left and right side are not identical (which is statistically not to be expected with the sample size we have) but the differences are small. We fitted the left and right side separately. The left side gives scale heights of $1.25 \pm 0.1 \mathrm{kpc}$ for the steep central part and $4.47 \pm 0.3 \mathrm{kpc}$ for the flanks. On the right side the values found are $1.30 \pm 0.1 \mathrm{kpc}$ and $4.60 \pm 0.3 \mathrm{kpc}$, respectively. We arrived at these values after several tries, e.g., by changing the bin size and, because of small number statistics there, the limits of $z$ to be included. For the fit of the central steep part we used a fitting interval of $[0.3,2.9]$ and for the flanks $[5,33]$. Given the similarity of the results we finally give one overall fit with $1.3 \pm 0.1 \mathrm{kpc}$ for the tight distribution and $4.5 \pm 0.3 \mathrm{kpc}$ for the extended distribution. The ratio of the mid-plane densities of the distributions is $N(0)_{\text {halo }} / N(0)_{\text {disk }}=0.16$.

The component with a steep $z$-distribution can be associated with the disk population, both the thin and thick disk. We tried a 3 component fit to separate thin and thick disk but did not arrive at sensible results. It is, from this data, not possible to separate thin and thick disk.

The analysis of the $z$-distribution statistics indicates the presence of (perhaps at least) two populations. The disk 


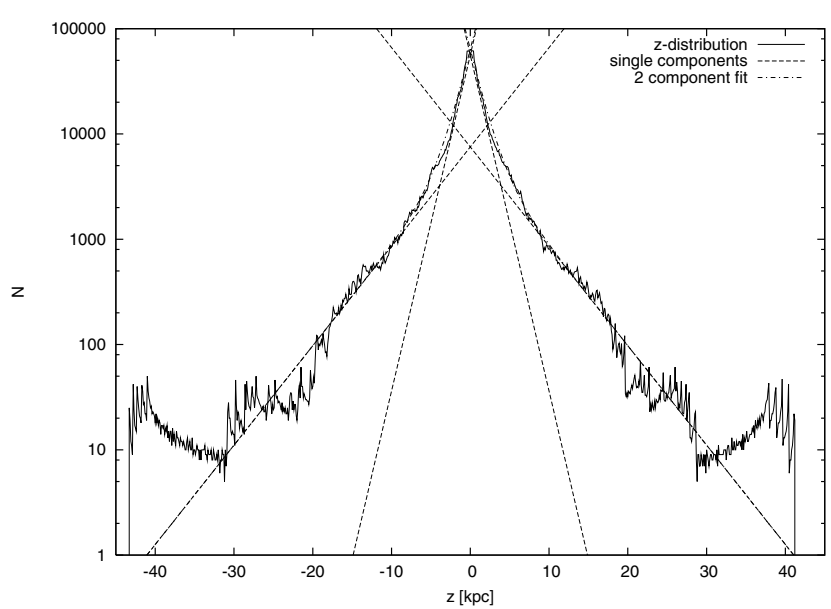

Fig. 6. The probability of the $z$-distance distribution of all stars of the sample, obtained by summing the individual $N(z)$ statistics for time steps of $1 \mathrm{Myr}$. The dashed lines show the two-component fit of the logarithmic distribution based on the scale heights $h=1.3 \pm 0.1 \mathrm{kpc}$ for the "disk component" and $h=4.5 \pm 0.3 \mathrm{kpc}$ for the "halo component".

population consists of stars which stay near the plane, while the halo population ventures to larger $z$. In order to explore a possible sample bias in relation with the scale height of the halo population we have made two tests with subsamples, as follows.

First, we selected those stars which at present are within the disk, i.e., those stars with $z_{\text {present }}<0.5 \mathrm{kpc}$. We made the $z$-statistics of this sample, as well as of the complement, and compared the two. The large scale $z$-distribution of the $z_{\text {present }}<0.5 \mathrm{kpc}$ group shows a pronounced peak near $z=$ $0 \mathrm{kpc}$ (which is of course what one expects). The $z$-distribution of the $z_{\text {present }}>0.5 \mathrm{kpc}$ group resembles the distribution of the whole sample but the mid-plane peak is missing.

The flanks of the $z$-statistics toward large $z$ of the two subsamples are very similar, indicating there is no sample selection in this sense, although the spatial distribution of the full sample is quite asymmetric (see Fig. 1).

In the subsample of the $z_{\text {present }}<0.5 \mathrm{kpc}$ group we find a slight separation of the thin and thick disk component leading to a scale height of $0.38 \pm 0.03 \mathrm{kpc}$ for the thin disk. This value is only slightly higher than that given by Chen et al. (2001). We note that our $z_{\text {present }}<0.5 \mathrm{kpc}$ sample contains 63 stars. Still, the separation of thin and thick disk is not well defined in our sample.

Second, we made a subdivision based on $z_{\max }<0.5 \mathrm{kpc}$. Here the small $z$ subsample is by definition restricted to stars staying close to the plane, while the $z_{\max }>0.5 \mathrm{kpc}$ subsample must contain predominantly halo stars. The small $z$ subsample (see Table 4 ) shows in a $\ln N$ versus $z$ diagram only a small high peak near $z=0 \mathrm{kpc}$ (by definition). This is further discussed in Sect. 5.

The large $z$ subsample shows a $z$-statistics quite similar to that of the full sample. It exhibits the scale height of $4.6 \pm 0.3 \mathrm{kpc}$ for the halo component, depending on the choice of bin size and the limits of $z$ to be included. That value is hardly different from the halo scale height obtained from the full sample. Even the disk component is present with the same

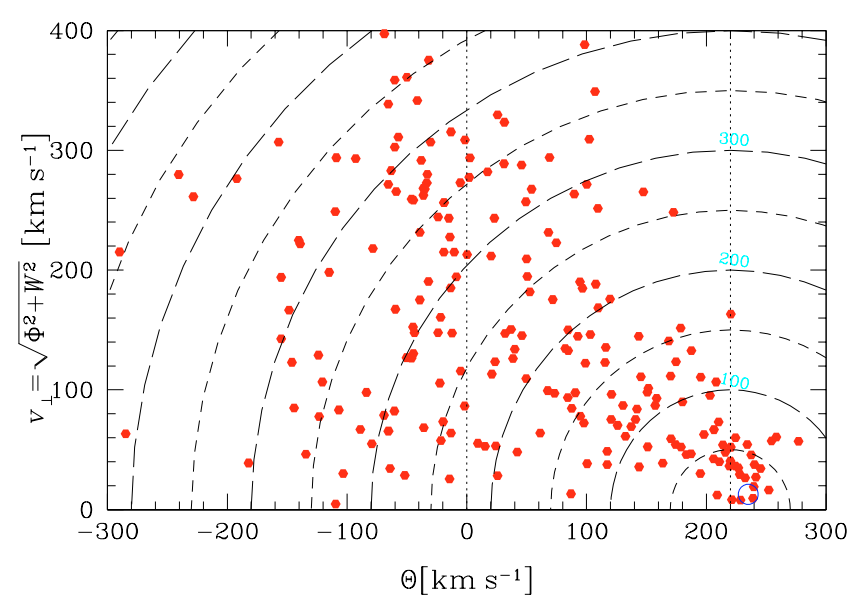

Fig. 7. Toomre diagram of $\Theta$ versus $v_{\text {perp }}$, the velocity perpendicular to the Galactic plane, showing the asymmetry in kinematics of the RR Lyrs. The dashed circles denote the peculiar velocity $v_{\text {pec }}=$ $\sqrt{\Phi^{2}+W^{2}+\left(\Theta-\Theta_{\mathrm{LSR}}\right)^{2}}$ in $\mathrm{km} \mathrm{s}^{-1}$. The open circle shows the parameters of the Sun.

values of scale height as found for our full sample of RR Lyrs, which is not surprising finding a scale height of the disk component of $1.3 \mathrm{kpc}$. The presence of a disk component in this sample shows that the disk population is dominated by stars with orbits reaching beyond $z=0.5 \mathrm{kpc}$.

We thus conclude that there is no significant sample bias in our RR Lyr star sample.

Altmann et al. (2004), dealing with sdB stars found with the same method a scale height of $0.9 \mathrm{kpc}$ for the disk component and a halo component with a scale height of $7 \mathrm{kpc}$ and Kaempf et al. (2005), considering RHB stars, give a scale height of $5 \mathrm{kpc}$ for the halo component.

\section{Sample statistics of $\Theta$ with ecc, nze, $[\mathrm{Fe} / \mathrm{H}]$}

\subsection{Circular velocity $\Theta$}

The RR Lyrs of our sample have a mean circular velocity of $\Theta=47 \mathrm{~km} \mathrm{~s}^{-1}$, but the stars cover a wide range (see also Table 2) with +277 and $-321 \mathrm{~km} \mathrm{~s}^{-1}$ as extreme velocities.

Looking at the histogram of $\Theta$ (Fig. 4) the most pronounced peak in the distribution is the one near $0 \mathrm{~km} \mathrm{~s}^{-1}$, marking the group which in a broad sense does not participate in galactic rotation.

The Toomre diagram (Fig. 7) allows to see the high asymmetry of our sample including many stars with peculiar velocities $v_{\text {pec }}>100 \mathrm{~km} \mathrm{~s}^{-1}$. This asymmetry is the so called asymmetric drift (for more on that see, e.g., the review by Majewski 1993). RR Lyrs with $\Theta \leq \Theta_{\text {LSR }}$ dominate our sample and the dispersion of data points is large. Only few stars with $\Theta \geq \Theta_{\text {LSR }}$ are present. This is clearly correlated with the high eccentricity of the orbits of most of the RR Lyrs as discussed in Sect. 3.2.

The Bottlinger diagrams (see Fig. 8) show the asymmetry of data points as well. At low values of $\Phi$ and $W$ the RR Lyrs do not concentrate near $\Theta_{\mathrm{LSR}}$ but have a great variety of kinematics. The kinematic dispersion of $W$ exceeds that of $\Phi$. The distribution of data points appears to be similar in the two panels. It seems to be symmetric in respect to both the $\Phi=0$ axis (upper panel) and the $W=0$ axis (lower panel). 
Table 2. Mean values of $U, V, W, \Theta, \Phi$, angular momentum, eccentricity and nze with dispersions for (subsets of) the sample.

\begin{tabular}{lrrrrrrrrrrrrrrrrr}
\hline \hline Subsample & $N$ & $\bar{U}$ & $\sigma_{U}$ & $\bar{V}$ & $\sigma_{V}$ & $\begin{array}{c}\bar{W} \\
{\left[\mathrm{~km} \mathrm{~s}^{-1}\right]}\end{array}$ & $\sigma_{W}$ & $\bar{\Theta}$ & $\sigma_{\Theta}$ & $\bar{\Phi}$ & $\sigma_{\Phi}$ & $\begin{array}{c}\bar{I}_{z} \\
{\left[\mathrm{kpc} \mathrm{km} \mathrm{s}^{-1}\right]}\end{array}$ & $\sigma_{I_{\mathrm{z}}}$ & $\overline{e c c}$ & $\sigma_{e c c}$ & $\overline{n z e}$ & $\sigma_{n z e}$ \\
\hline all & 217 & -3 & 158 & +47 & 125 & +3 & 85 & +47 & 123 & +5 & 159 & +395 & 1027 & 0.61 & 0.30 & 0.63 & 0.79 \\
{$[\mathrm{Fe} / \mathrm{H}]>-0.5$} & 20 & -2 & 48 & +206 & 31 & -3 & 38 & +204 & 32 & +3 & 53 & +1718 & 356 & 0.19 & 0.13 & 0.09 & 0.11 \\
{$[\mathrm{Fe} / \mathrm{H}]>-1$} & 46 & 0 & 82 & +165 & 93 & -3 & 53 & +168 & 90 & +4 & 82 & +1436 & 810 & 0.32 & 0.28 & 0.32 & 0.67 \\
{$[\mathrm{Fe} / \mathrm{H}] \leq-1$} & 169 & -4 & 173 & +15 & 113 & +5 & 92 & +14 & 110 & +6 & 175 & +113 & 890 & 0.69 & 0.25 & 0.71 & 0.79 \\
{$[\mathrm{Fe} / \mathrm{H}] \leq-1.5$} & 86 & -25 & 177 & -13 & 114 & +5 & 97 & -13 & 108 & +27 & 181 & -105 & 880 & 0.72 & 0.23 & 0.67 & 0.61 \\
$e c c \leq 0.45^{a}$ & 58 & -15 & 54 & +195 & 40 & -19 & 64 & +196 & 40 & +19 & 51 & +1644 & 395 & 0.22 & 0.12 & 0.19 & 0.35 \\
$e c c>0.45$ & 149 & +1 & 186 & -2 & 92 & +11 & 91 & -3 & 88 & 0 & 188 & -20 & 722 & 0.79 & 0.17 & 0.81 & 0.85 \\
$P<0.35 \mathrm{~d}$ & 20 & -37 & 133 & +44 & 116 & +33 & 100 & +44 & 115 & +39 & 134 & +387 & 990 & 0.59 & 0.30 & 0.64 & 0.49 \\
$0.35<P<0.55 \mathrm{~d}$ & 103 & +2 & 164 & +68 & 137 & +2 & 76 & +68 & 135 & +1 & 165 & +583 & 1131 & 0.57 & 0.32 & 0.53 & 0.75 \\
$P>0.55 \mathrm{~d}$ & 91 & -1 & 157 & +22 & 108 & -1 & 92 & +21 & 106 & +3 & 159 & +170 & 861 & 0.68 & 0.26 & 0.74 & 0.87 \\
\hline
\end{tabular}

${ }^{a}$ Without seven stars having retrograde orbits.

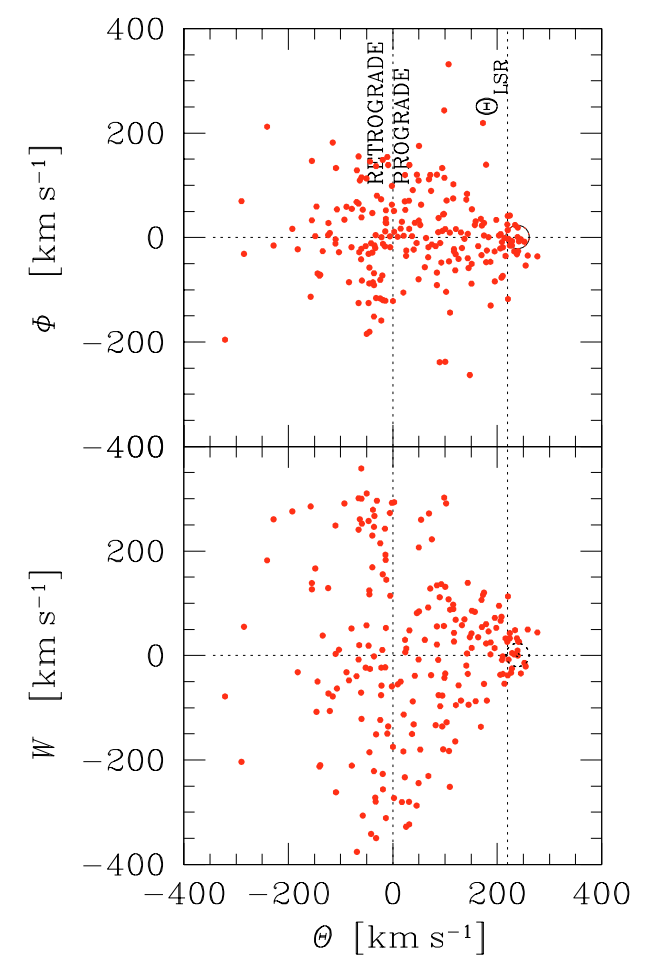

Fig. 8. Bottlinger and $\Theta-W$ diagrams show the orbital velocity of the RR Lyrae stars plotted against the velocity towards the Galactic centre $(\Phi$, top $)$ and perpendicular to the Galactic plane $(W$, bottom). A circle indicates the Solar values.

\subsection{Discussion of sample metallicities in relation with orbits and periods}

Given the orbit parameters, it is worthwhile to relate them with other parameters of RR Lyrs. For that, we have divided our sample in subgroups in various ways (Table2). We used non kinematic selection criteria as metallicity and period but the kinematic criterion eccentricity as well. We choose as cuts $[\mathrm{Fe} / \mathrm{H}]>-0.5,>-1, \leq-1$, and $\leq-1.5$, for $e c c \leq 0.45$ and $>0.45$, and for period $P<0.35 \mathrm{~d}, 0.35<P<0.55 \mathrm{~d}$ and $P>0.55 \mathrm{~d}$.

Metallicity is not a kinematic parameter. Yet, metallicity gives no clear division between disk and halo stars. Stars with
$[\mathrm{Fe} / \mathrm{H}] \geq-1$ may be members of the disk group in view of their $\bar{\Theta}$ (in particular those with $[\mathrm{Fe} / \mathrm{H}] \geq-0.5$ ). But the velocity dispersion $\sigma_{\Theta} \simeq 90 \mathrm{~km} \mathrm{~s}^{-1}$ is larger than expected for a pure disk population. So some of the RR Lyrs with $[\mathrm{Fe} / \mathrm{H}] \geq-1$ can be regarded to be halo stars.

The more metal-poor RR Lyrs with $[\mathrm{Fe} / \mathrm{H}] \leq-1$ are candidates for halo stars. This is corroborated by the result of $\bar{\Theta}=14 \mathrm{~km} \mathrm{~s}^{-1}, \sigma_{\Theta}=110 \mathrm{~km} \mathrm{~s}^{-1}, \overline{e c c}=0.69$ and $\overline{n z e}=0.71$ for this group. The velocity values of $\bar{U}, \bar{V}$ and $\bar{U}$ for the metal poor part of our sample are similar to several published halo samples (see Martin \& Morrison 1998, their Table 5) even if in their works the cuts for halo stars are set at more metal poor levels. Therefore we give the results for RR Lyrs with $[\mathrm{Fe} / \mathrm{H}] \leq-1.5$, too. For this group our results shows to be in good agreement with results of previous investigations (see Martin \& Morrison 1998; Chen 1999). This metal-poor group of RR Lyrs has $\bar{\Theta}=-13 \mathrm{~km} \mathrm{~s}^{-1}$, i.e., a mean retrograde rotation with respect to the $\mathrm{LSR}^{2}$.

Orbit eccentricity supposedly divides between younger and older stars (more gravitational interactions of disk stars lead to larger deviations from the originally circular orbits). The subdivision at ecc $=0.45$ shows the expected correlations with kinematic parameters (see Table 2; notably $\bar{I}_{\mathrm{z}}$ ). This subdivision does not correlate in a pronounced way with metallicity.

Note that we did not include 7 stars with retrograde orbits in the subgroup with ecc $\leq 0.45$ (Table 2). These 7 have a mean circular velocity $\bar{\Theta}=-165 \mathrm{~km} \mathrm{~s}^{-1}$ with dispersion of $\sigma_{\Theta}=56 \mathrm{~km} \mathrm{~s}^{-1}$. Also Chiba \& Yoshii (1997) report the presence of halo stars with low eccentricity orbits. Both results show that low eccentricity does not always mean that a star is a disk member. The 58 RR Lyrs are good candidates for disk stars (thin and thick disk).

Pulsation periods are related with the mass of the RR Lyr, which is likely related with the development history of the star.

The groups with pulsation period $P<0.35 \mathrm{~d}$ and $P>$ $0.55 \mathrm{~d}$ show both lower abundances than the large third group of periods $0.35<P<0.55 \mathrm{~d}$. It has the largest abundance and the largest dispersion $\sigma_{[\mathrm{Fe} / \mathrm{H}]}$. It is not a uniform

\footnotetext{
${ }^{2}$ Note that results for $\Theta$ as given in the literature are sometimes based on $\Theta_{\odot}=0$, sometimes on $\Theta_{\odot}=220 \mathrm{~km} \mathrm{~s}^{-1}$.
} 
Table 3. $[\mathrm{Fe} / \mathrm{H}]$ and Period with their dispersions for the $217 \mathrm{RR}$ Lyrae stars of the sample.

\begin{tabular}{lrrrrr}
\hline \hline & $N$ & & & & \\
\cline { 5 - 6 } Subsample & & $\overline{\mathrm{Fe} / \mathrm{H}]}$ & $\sigma_{[\mathrm{Fe} / \mathrm{H}]}$ & $\begin{array}{c}\text { Period } \\
\mathrm{d}\end{array}$ & $\sigma_{\text {Period }}$ \\
\hline all & 217 & -1.33 & 0.51 & 0.51 & 0.12 \\
{$[\mathrm{Fe} / \mathrm{H}]>-0.5$} & 20 & -0.28 & 0.16 & 0.44 & 0.06 \\
{$[\mathrm{Fe} / \mathrm{H}]>-1$} & 46 & -0.55 & 0.30 & 0.44 & 0.09 \\
{$[\mathrm{Fe} / \mathrm{H}] \leq-1$} & 169 & -1.54 & 0.32 & 0.53 & 0.11 \\
{$[\mathrm{Fe} / \mathrm{H}] \leq-1.5$} & 86 & -1.80 & 0.23 & 0.56 & 0.12 \\
$e c c \leq 0.45^{a}$ & 58 & -0.90 & 0.57 & 0.47 & 0.09 \\
$e c c>0.45$ & 149 & -1.48 & 0.36 & 0.53 & 0.11 \\
$P<0.35 \mathrm{~d}$ & 20 & -1.39 & 0.48 & 0.29 & 0.07 \\
$0.35<P<0.55 \mathrm{~d}$ & 103 & -1.11 & 0.51 & 0.47 & 0.05 \\
$P>0.55 \mathrm{~d}$ & 91 & -1.58 & 0.40 & 0.62 & 0.06 \\
\hline
\end{tabular}

${ }^{a}$ Not including seven stars having retrograde orbits.
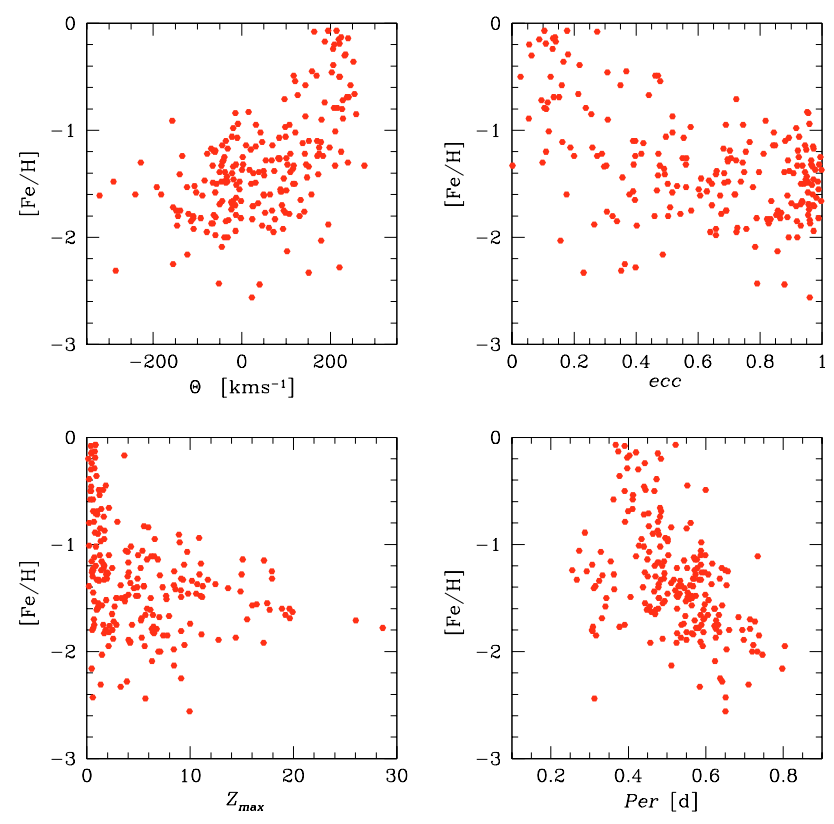

Fig. 9. The plot shows metallicity $[\mathrm{Fe} / \mathrm{H}]$ versus circular velocity $\Theta$ (upper left panel), ecc (upper right), nze (lower left) and period (lower right panel). RR Lyrs with $[\mathrm{Fe} / \mathrm{H}]>-1$ show more disk-like orbits, but more metal-poor stars present both, disk-like orbits and halo-like orbits having smaller $\Theta$, large eccentricity ecc and large normalised $z$-distance nze. With $[\mathrm{Fe} / \mathrm{H}]$ versus period (lower right panel), note that there are 4 groups of stars. For the discussion see Sect. 4.2.

subsample of RR Lyrs. That this group is so different is also visible in Table 2. The dispersion in $U, V, \Theta, \Phi, I_{\mathrm{z}}$ and ecc is larger in this subsample than in the two other period groups. This fact can be noted as well in Fig. 9 (lower right panel).

The group with periods of $<0.35 \mathrm{~d}$ contains the RRc stars with abundances of $-2<[\mathrm{Fe} / \mathrm{H}]<-1$. The metallicity of the second period group ranges from solar to $[\mathrm{Fe} / \mathrm{H}]$ of $\simeq-2$. These are RRab stars. Note that the period increases in this group toward decreasing metallicity. Most of the RR Lyrs of our sample are in this group. It combines RR Lyrs with a large dispersion in metallicity and various orbit parameters, as can be recognised in Table 3, probably representing different ages.
The third group having periods $\simeq 0.6 \mathrm{~d}$ are $\mathrm{RRab}$ stars as well but with lower abundances. This group is the field RR Lyr equivalent to the RR Lyrs in Oosterhoff II clusters. This last group is a very small subsample of RR Lyrs with relative long periods and metallicities $[\mathrm{Fe} / \mathrm{H}]<-1.6$.

All parameters are considered in Table 3. RR Lyrs with ecc $>0.45$ have lower abundances $(\overline{[\mathrm{Fe} / \mathrm{H}]}=-1.48)$ than those with $e c c<0.45(\overline{[\mathrm{Fe} / \mathrm{H}]}=-0.90)$, as we expected. Like in Table 2 we did not include 7 RR Lyrs with retrograde orbits having ecc $<0.45$. We did so because these stars do not fit into the group of low eccentricity stars which are candidate disk stars. This group of retrograde RR Lyrs has a low metallicity of $\overline{[\mathrm{Fe} / \mathrm{H}]}=-1.85$.

Figure 9 shows the data of Tables 2 and 3 in graphical form: metallicity $[\mathrm{Fe} / \mathrm{H}]$ versus orbital velocity $\Theta$, ecc, nze, and period. Dividing the RR Lyrs in metal-poor and metal-rich groups as Layden (1995), we also find that velocities for RR Lyrs with $[\mathrm{Fe} / \mathrm{H}]>-1$ reveal a distribution different than those with lower metallicities. High metallicity stars with more disk-like circular velocities $\Theta$ cluster both at low ecc and nze. But the separation is not a strict one. Even some data points with very low abundance $[\mathrm{Fe} / \mathrm{H}] \leq-1.5$ show $\Theta \simeq 200 \mathrm{~km} \mathrm{~s}^{-1}$, ecc $<0.4$ and $n z e<0.2$. Altmann $\&$ de Boer (2000) found the same fact using a much smaller sample of RR Lyrs.

These findings are similar to those by Chiba \& Yoshii (1998) who report that even stars with very low metallicity may have "nearly circular orbits with ecc $<0.4$ ". High metallicity does indicate disk-like kinematics, but low metallicity does all by itself not give sufficient information about the orbit shape. An eccentricity of $<0.4$ is alone not a sufficient criterion for population. Chiba \& Beers (2000) find in their investigation of metal-poor stars "a remarkable discontinuity of the rotational properties of the Galaxy" at $[\mathrm{Fe} / \mathrm{H}] \simeq-1.7$ (see also their Fig. 3). Our sample of RR Lyrs does not confirm this. We find a continuous distribution in $\Theta$,ecc and nze in the metallicity range $-2<[\mathrm{Fe} / \mathrm{H}]<-1$. But we note that in a sample of 163 metal-poor halo stars chances are non-zero to find one with an orbit rather co-planar with the Milky Way disk.

\section{Discussion of results}

We calculated the orbits of the RR Lyrs of our sample and derived the $z$-distribution statistics. This led to scale heights for a disk group of RR Lyrs $\left(h_{\text {disk }} \simeq 1.3 \mathrm{kpc}\right.$ ) and for a halo group ( $h_{\text {halo }} \simeq 4.5 \mathrm{kpc}$ ). However, analysing for each star the parameters $\Theta$, ecc, nze, and $[\mathrm{Fe} / \mathrm{H}]$ it became clear that all parameters are needed to classify a given star as a genuine disk or halo star. Of our 217 stars, 63 are truly disk like, 154 are halo like.

In our sample we have 87 RR Lyrs with retrograde orbits. We compared the characteristics of these stars with the prograde ones. Among the retrograde part of our sample there are no stars with metallicity $[\mathrm{Fe} / \mathrm{H}]>-0.9$ (see Fig. 9 upper left panel). These retrograde rotating RR Lyrs are not separated from the rest of the sample in respect to $z_{\max }$ or period, but their group values of $\overline{e c c}=0.79, \sigma_{e c c}=0.20, \overline{n z e}=0.79$ and $\sigma_{\text {nze }}=0.79$ are higher than the values for prograde halo candidates of about the same metallicity. We again conclude that most of the RR Lyrs of our sample are halo stars. 


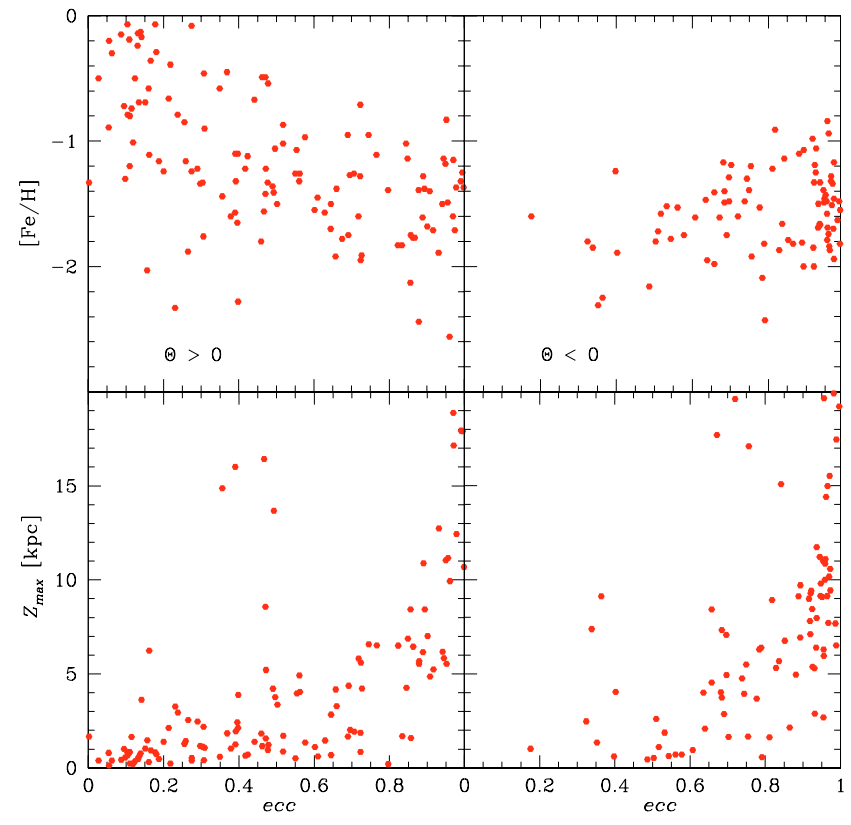

Fig. 10. Plots ecc against $[\mathrm{Fe} / \mathrm{H}]$ and $Z_{\max }$. On the left side only RR Lyrs with prograde orbits $(\Theta>0)$ are plotted, on the right side only those with retrograde orbits $(\Theta<0)$. The eccentricities of the prograde RR Lyrs spread over the hole range of ecc while the retrograde ones show foremost higher eccentricities.

A different way of looking at the data is given in Fig. 10. There $[\mathrm{Fe} / \mathrm{H}]$ and $Z_{\max }$ are plotted against ecc for the prograde and retrograde RR Lyrs. Clearly, the continuous distribution indicated above is present for the prograde set, while the retrograde stars rather are metal poor and have orbits with predominantly larger eccentricity. In the retrograde set there are only stars with $[\mathrm{Fe} / \mathrm{H}]<-0.8$ and $e c c>0.45$. We conclude from this that the prograde set does contain a good fraction of higher metallicity low-eccentricity orbit stars, in short younger RR Lyrs, while the retrograde set has only those with characteristics not like that of a younger galactic disk.

We took a special look at the stars not reaching beyond $z_{\max }=0.5 \mathrm{kpc}$ These stars are listed in Table 4. Among these stars are two with a much smaller metallicity than the rest and they turn out to have very eccentric orbits. These are RR Lyr itself, and V338 Pup whose orbit is retrograde. Thus 2 of 16 stars staying close to the plane turn out not to have disk-like orbits and thus belong to the halo population.

Chiba \& Beers (2000) published a list of likely metal-weak thick disk stars including $3 \mathrm{RR}$ Lyrs using as criterium $[\mathrm{Fe} / \mathrm{H}]$ $\leq-1, e c c \leq 0.2$ and $183<\Theta<225 \mathrm{~km} \mathrm{~s}^{-1}$. In our sample we found 7 such RR Lyrs: the three found by Chiba \& Beers (2000) plus TZ Aqr, RX Eri, V690 Sco and AT Ser. We confirm that there are metal-weak stars with classical disk orbits, which may be part of a metal-weak thick disk or in two cases (V494 Sco and V690 Sco) even thin disk star candidates having a $z_{\max }<$ $0.5 \mathrm{kpc}$.

\section{Halo distribution of RR Lyrae stars}

The spatial distribution of RR Lyrs and other HB-like stars has been investigated several times based on diverse samples.
Table 4. 16 RR Lyrs staying close to the plane $\left(z_{\max }<0.5 \mathrm{kpc}\right)$.

\begin{tabular}{lrrrrrr}
\hline \hline Name & {$[\mathrm{Fe} / \mathrm{H}]$} & $\begin{array}{r}\Theta \\
{\left[\mathrm{km} \mathrm{s}^{-1}\right]}\end{array}$ & $\begin{array}{r}R_{\mathrm{a}} \\
{[\mathrm{kpc}]}\end{array}$ & $\begin{array}{r}R_{\mathrm{p}} \\
{[\mathrm{kpc}]}\end{array}$ & $\begin{array}{r}z_{\max } \\
{[\mathrm{kpc}]}\end{array}$ & $e c c$ \\
\hline SW And & -0.24 & 206.0 & 9.43 & 7.25 & 0.47 & 0.13 \\
AA CMi & -0.15 & 219.1 & 10.45 & 8.77 & 0.43 & 0.09 \\
UY Cyg & -0.80 & 226.6 & 9.61 & 7.70 & 0.23 & 0.11 \\
DM Cyg & -0.14 & 240.1 & 10.59 & 8.11 & 0.48 & 0.13 \\
DX Del & -0.39 & 206.4 & 9.84 & 6.31 & 0.23 & 0.22 \\
FW Lup & -0.20 & 209.2 & 8.24 & 7.38 & 0.13 & 0.06 \\
RR Lyr & -1.39 & 109.4 & 18.37 & 2.08 & 0.21 & 0.80 \\
CN Lyr & -0.58 & 245.3 & 10.64 & 7.70 & 0.32 & 0.16 \\
KX Lyr & -0.46 & 203.2 & 10.86 & 5.76 & 0.41 & 0.31 \\
AV Peg & -0.08 & 163.6 & 8.63 & 4.91 & 0.39 & 0.27 \\
CG Peg & -0.50 & 221.4 & 8.67 & 8.21 & 0.39 & 0.03 \\
AR Per & -0.30 & 232.4 & 10.26 & 9.06 & 0.40 & 0.06 \\
HH Pup & -0.50 & 220.2 & 10.01 & 7.81 & 0.35 & 0.12 \\
V338 Pup & -2.16 & -123.2 & 9.36 & 3.24 & 0.46 & 0.49 \\
V494 Sco & -1.01 & 210.9 & 8.54 & 6.73 & 0.21 & 0.12 \\
V690 Sco & -1.16 & 198.1 & 8.49 & 5.81 & 0.48 & 0.19 \\
\hline
\end{tabular}

In a pioneering study, Plaut (1965) derived a scale height of RR Lyr stars in the solar vicinity of $\simeq 2.3 \mathrm{kpc}$. In his review Majewski (1993) reports scale heights for the "intermediate population II”, being $\simeq 1.5 \mathrm{kpc}$ for RR Lyrs. In almost all cases, the studies used photometric distances perhaps together with radial velocity information. Proper motions have thus far been included in limited cases only.

The vertical scale height derived in this paper from the orbit statistics can be used for a comparison with results from other studies.

Kinman et al. (1994) used BHB stars (with colours between the blue edge of the RR Lyr strip and the intersection of the $\mathrm{HB}$ with the main sequence, so rather HBA stars) to derive the structure of the halo from statistically complete samples. They assume a (spherical) radial distribution of the form

$\rho=\rho_{0} R_{\mathrm{gal}}^{-3.5}$

and find that the distribution of their HBA stars agrees with that. We have fitted our $z$-distribution to the barometric formula (Eq. (2)) and arrived at a scale height of $\simeq 5 \mathrm{kpc}$ (see Fig. 6). Transforming the run of densities of a radial distribution into an exponential vertical distribution at the location of the Sun $(\varpi=8.5 \mathrm{kpc})$ we find that the radial and the barometric relation (with $h=5 \mathrm{kpc}$ ) give a run of densities $N(z)$ which, for $5<z<$ $20 \mathrm{kpc}$, deviate from each other by only $20 \%$ (the barometric formula being a bit steeper). Thus our result from RR Lyr star orbits shows a spatial distribution similar to the distribution of HBA stars from samples by Kinman et al. (1994).

The mean space density of RR Lyrs in the solar neighbourhood has been derived by Amrose \& Mckay (2001). They arrive at different values for different solar distances but their overall result is fairly summarised by $\rho=5 \pm 1$ stars $\mathrm{kpc}^{-3}$. They also derive a vertical distribution and give fits with the barometric formula allowing scale heights between 0.75 and $1.8 \mathrm{kpc}$ (using a single exponential fit). However, from their Fig. 5 it is clear that the RR Lyrs show a steeper distribution at low $z$ with $h \simeq 1 \mathrm{kpc}$, similar to our result, and a flatter one further out of $h>2 \mathrm{kpc}$. 
Our data cannot be used to find an absolute midplane density. However, one could use the ratio of $N(0)_{\text {halo }} / N(0)_{\text {diskRR Lyrs }}=0.16$ together with the Amrose \& Mckay value of $\rho=5 \pm 1$ stars kpc ${ }^{-3}$. Furthermore, the midplane ratio may be combined with the scale heights derived for the disk and halo components to get the ratio of all RR Lyrs in these components. The procedure is similar to that used by de Boer (2004) for sdB stars. We so find that the Milky Way has about half as many halo RR Lyrs than disk RR Lyrs. Since the midplane density ratio probably is still affected by systematic effects and the scale heights are, among various studies, only just consistent, the indicated total RR Lyr star ratio has to be regarded as a crude value.

The data from the Sloan Digital Sky Survey (SDSS) revealed numerous HB stars also at very large distances from the Milky Way disk (see, e.g., Yanny et al. 2000; Sirko et al. 2004). Vivas et al. (2001), using data from the QUEST survey for RR Lyrs, find an over-density of RR Lyrs at $50 \mathrm{kpc}$ from the Sun. They surmise this over-density is part of the tidal stream associated with the Sagittarius dwarf spheroidal galaxy. From these new survey results we conclude that the halo is, on a large scale, apparently rather inhomogeneous so that RR Lyr distribution values will show to vary in different directions and at different distances.

\section{Conclusions}

RR Lyrs exist in the Milky Way as part of the disk and part of the halo. We separated the halo component from disk candidates using the shape of their orbits. Of 217 RR Lyrs, 154 or $71 \%$ are member of the halo population. Therein we find a retrograde rotating halo component of $87 \mathrm{RR}$ Lyrs or more than half of our halo group. The (vertical) spatial distribution found with our orbit statistics method is, out to $z=20 \mathrm{kpc}$, similar to that found in other studies using classical methods. We show that there are RR Lyrs staying in the disk but which are member of the halo population.

Acknowledgements. The orbit programme was put at the disposal of the Bonn group by Michael Odenkirchen. We thank Martin Altmann for providing several plotting programs as well as constructive criticism and Torsten Kaempf and Michael Geffert for helpful discussions and the referee, Tim Beers for suggestions that improved the paper.

\section{References}

Altmann, M., \& de Boer, K. S. 2000, A\&A, 353, 135

Altmann, M., Edelmann, H., \& de Boer, K. S. 2004, A\&A, 414, 181

Amrose, S., \& Mckay, T. 2001, ApJ, 560, L151

Beers, T. C., Chiba, M., Joshii, Y., et al. 2000, AJ, 119, 2688

de Boer, K. S., Tucholke, H.-J., \& Schmidt, J. H. K. 1997a, A\&A, 317, L23

de Boer, K. S., Aguilar Sánchez, Y., Altmann, M., et al. 1997b, A\&A, 327,577

de Boer, K. S., 2004, A\&A, 419, 527

Chen, B., 1999, A\&A, 344, 494

Chen, B., Stoughton, C., Smith, J. A., et al. 2001, ApJ, 553, 184

Chiba, M., \& Yoshii, Y. 1997, AJ, 490, L73

Chiba, M., \& Yoshii, Y. 1998, AJ, 115, 168

Chiba, M., \& Beers, T. C. 2000, AJ, 119, 2843

ESA 1997, The Hipparcos and Tycho Catalogues, ESA SP-1200

Fernley, J., \& Barnes, T. G. 1997, A\&AS, 125, 313

Fernley, J., Barnes, T. G., Skillen, I., et al. 1998, A\&A, 330, 515

Heber, U. 1986, A\&A, 155, 33

Kaempf, T. A., de Boer, K. S., \& Altmann, M. 2005, A\&A, 432, 879

Kinman, T., Suntzeff, N. B., \& Kraft, R. P. 1994, AJ, 108, 1722

Lasker, B. M., Russel, J. N., \& Jenkner, H. 1996, The HST Guide Star Catalogue, Version 1.2

Layden, A. C. 1994, AJ, 108, 1016

Layden, A. C. 1995, AJ, 110, 2312

Maintz, G. 2005, A\&A, 442, 381

Martin, J. C., \& Morrison, H. L. 1998, AJ, 116, 1724

Majewski, S. R. 1993, ARA\&A, 31, 575

Moehler, S., de Boer, K. S., \& Heber, U. 1990, A\&A, 239, 265

Odenkirchen, M., \& Brosche, P. 1992, AN, 313, 69

Ojha, D. K., Bienayme, O., Robin, A. C., et al. 1994, A\&A, 284, 810

Plaut, L. 1965, in Galactic Structure, ed. A. Blaauw, \& M. Schmidt (Univ. Chicago Press), 267

Saffer, R. A., Bergeron, P., Koester, D., et al. 1994, ApJ, 432, 351

Sirko, E., Goodman, J., Knapp, G., R, et al. 2004, AJ, 127, 899

Theisen, A., Moehler, S., Heber, U., et al. 1993, A\&A, 273, 524

Villeneuve, B., Wesemael, F., Fontaine, G., et al. 1995, ApJ, 446, 646

Vivas, A. K., Zinn, R., Andrews, P., et al. 2001, ApJ, 554, L33

Yanny, B., Newberg, H. J., Kent, S., et al. 2000, ApJ, 540, 825 
G. Maintz and K. S. de Boer: RR Lyrae stars: kinematics, orbits and z-distribution, Online Material p 1

\section{Online Material}


G. Maintz and K. S. de Boer: RR Lyrae stars: kinematics, orbits and z-distribution, Online Material p 2

Table 1. Positions, velocities (given in the Galactic euclidic system $X Y Z U V W$ and $\Theta, \Phi)$ and orbital data $\left(R_{\mathrm{a}}, R_{\mathrm{p}}, z_{\max }\right.$, normalised $z$-extent, $n z e$ and eccentricy ecc) of the stars of our sample.

\begin{tabular}{|c|c|c|c|c|c|c|c|c|c|c|c|c|c|}
\hline Name & $\begin{array}{r}X \\
{[\mathrm{kpc}]}\end{array}$ & $Y$ & $Z$ & $\begin{array}{c}U \\
{\left[\mathrm{~km} \mathrm{~s}^{-1}\right]}\end{array}$ & $\bar{V}$ & $W$ & $\Phi$ & $\Theta$ & $\begin{array}{c}R_{\mathrm{a}} \\
{[\mathrm{kpc}]}\end{array}$ & $R_{\mathrm{p}}$ & $z_{\max }$ & $\begin{array}{r}n z e \\
- \\
\end{array}$ & $\begin{array}{r}e c c \\
-\end{array}$ \\
\hline SW And & -8.70 & 0.42 & -0.30 & 46.8 & 204.0 & -20.9 & -37.0 & 206.0 & 9.43 & 7.25 & 0.47 & 0.05 & 0.13 \\
\hline XX And & -9.09 & 0.74 & -0.41 & -193.3 & 1.5 & -120.9 & 192.8 & -14.1 & 15.94 & 0.22 & 9.44 & 0.83 & 0.97 \\
\hline AT And & -8.78 & 0.79 & -0.27 & 184.8 & 3.7 & -105.1 & -183.8 & 20.2 & 13.89 & 0.36 & 11.04 & 1.91 & 0.95 \\
\hline CI And & -9.73 & 1.23 & -0.55 & -3.0 & 239.8 & -31.7 & 33.0 & 237.6 & 12.69 & 9.35 & 1.03 & 0.08 & 0.15 \\
\hline DR And & -9.60 & 1.50 & -1.01 & -113.7 & 12.6 & -18.2 & 114.3 & -5.1 & 11.46 & 0.08 & 7.68 & 1.22 & 0.99 \\
\hline WY Ant & -8.55 & -0.96 & 0.39 & 281.7 & -1.4 & 4.6 & -279.8 & -32.8 & 20.37 & 0.48 & 11.01 & 0.69 & 0.95 \\
\hline TY Aps & -7.59 & -1.01 & -0.25 & -51.9 & 25.4 & 139.0 & 48.0 & 32.0 & 8.00 & 1.17 & 6.58 & 1.65 & 0.74 \\
\hline XZ Aps & -7.46 & -1.32 & -0.55 & 78.7 & -8.9 & 73.4 & -76.0 & -22.5 & 8.57 & 0.33 & 5.38 & 1.17 & 0.93 \\
\hline BS Aps & -7.04 & -1.32 & -0.53 & -94.3 & 264.1 & -36.1 & 44.2 & 277.0 & 13.03 & 6.95 & 1.12 & 0.09 & 0.30 \\
\hline SW Aqr & -7.87 & 0.79 & -0.62 & 308.1 & -44.2 & 52.2 & -311.0 & -13.1 & 23.70 & 0.21 & 19.90 & 1.90 & 0.98 \\
\hline SX Aqr & -7.76 & 1.18 & -0.94 & 361.4 & -124.3 & 128.7 & -375.9 & -68.7 & 51.04 & 0.99 & 14.40 & 0.29 & 0.96 \\
\hline TZ Aqr & -7.70 & 1.08 & -1.31 & -20.1 & 187.8 & 0.9 & 45.9 & 183.2 & 8.44 & 5.62 & 1.39 & 0.17 & 0.20 \\
\hline BO Aqr & -7.92 & 0.84 & -1.68 & 99.7 & 120.7 & 9.9 & -86.4 & 130.5 & 9.18 & 3.40 & 1.82 & 0.20 & 0.46 \\
\hline BR Aqr & -8.36 & 0.53 & -1.18 & 9.8 & 186.8 & -46.6 & 2.0 & 187.1 & 8.46 & 6.72 & 1.65 & 0.20 & 0.11 \\
\hline BV Aqr & -7.92 & 0.36 & -0.86 & -81.8 & 54.1 & 175.4 & 84.2 & 50.4 & 8.97 & 3.23 & 8.58 & 2.21 & 0.47 \\
\hline CP Aqr & -7.68 & 0.93 & -0.76 & 107.5 & 168.3 & -24.5 & -86.5 & 180.0 & 9.34 & 4.93 & 1.07 & 0.12 & 0.31 \\
\hline DN Aqr & -8.12 & 0.27 & -1.22 & -292.2 & 8.2 & 99.0 & 292.3 & -1.6 & 23.70 & 0.04 & 19.22 & 1.55 & 1.00 \\
\hline AA Aql & -7.59 & 0.85 & -0.58 & 50.8 & 139.0 & 7.0 & -35.0 & 143.8 & 7.83 & 3.77 & 0.60 & 0.08 & 0.35 \\
\hline V341 Aql & -7.87 & 0.64 & -0.36 & -78.6 & 116.5 & -143.8 & 87.8 & 109.7 & 9.48 & 3.40 & 5.21 & 0.65 & 0.47 \\
\hline S Ara & -7.70 & -0.24 & -0.18 & 176.4 & 102.0 & 44.3 & -179.5 & 96.5 & 11.49 & 1.85 & 0.85 & 0.07 & 0.72 \\
\hline IN Ara & -6.91 & -0.60 & -0.49 & 127.0 & 106.1 & 133.2 & -135.8 & 94.6 & 10.13 & 1.85 & 4.37 & 0.51 & 0.69 \\
\hline MS Ara & -6.98 & -0.54 & -0.42 & -15.1 & -47.5 & -125.1 & 18.7 & -46.2 & 7.15 & 1.08 & 4.76 & 0.99 & 0.74 \\
\hline X Ari & -8.91 & 0.08 & -0.35 & 23.0 & -52.0 & -16.3 & -23.5 & -51.8 & 9.01 & 1.05 & 0.57 & 0.06 & 0.79 \\
\hline RW Ari & -9.85 & 0.78 & -1.35 & 67.8 & 169.6 & 4.0 & -54.2 & 174.5 & 10.80 & 6.36 & 1.44 & 0.13 & 0.26 \\
\hline TZ Aur & -9.96 & 0.08 & 0.56 & -72.2 & 208.7 & -76.8 & 73.9 & 208.1 & 13.07 & 8.05 & 2.95 & 0.23 & 0.24 \\
\hline RS Boo & -8.31 & 0.23 & 0.73 & 21.3 & 251.7 & -8.0 & -14.2 & 252.2 & 11.57 & 8.28 & 0.94 & 0.08 & 0.17 \\
\hline ST Boo & -8.13 & 0.58 & 1.00 & 29.2 & 139.1 & 72.8 & -19.1 & 140.8 & 8.21 & 4.36 & 2.19 & 0.27 & 0.31 \\
\hline TV Boo & -8.41 & 0.50 & 1.20 & 134.1 & 32.1 & -22.9 & -132.0 & 40.1 & 10.58 & 0.68 & 5.67 & 0.77 & 0.88 \\
\hline TW Boo & -8.30 & 0.58 & 1.20 & 254.1 & -36.8 & -12.1 & -256.1 & -18.9 & 17.61 & 0.25 & 10.57 & 0.84 & 0.97 \\
\hline UU Boo & -7.87 & 0.96 & 1.84 & 246.9 & -139.6 & 133.2 & -261.9 & -108.8 & 23.16 & 3.22 & 17.10 & 1.08 & 0.76 \\
\hline UY Boo & -8.05 & -0.04 & 1.16 & 233.1 & 24.3 & 69.3 & -233.2 & 23.0 & 15.66 & 0.32 & 9.94 & 0.94 & 0.96 \\
\hline AE Boo & -8.05 & 0.15 & 0.86 & 88.4 & 36.9 & 90.8 & -87.7 & 38.5 & 9.73 & 0.63 & 5.53 & 0.89 & 0.88 \\
\hline $\mathrm{U} \mathrm{Cae}$ & -9.23 & -1.32 & -1.22 & -84.8 & 168.2 & 139.3 & 60.0 & 178.6 & 11.51 & 8.30 & 6.24 & 0.63 & 0.16 \\
\hline $\mathrm{U} Y \mathrm{Ya}$ & -9.49 & 0.77 & 0.75 & -33.8 & 120.4 & -22.0 & 43.4 & 117.3 & 9.81 & 3.47 & 0.96 & 0.11 & 0.48 \\
\hline RW Cnc & -9.72 & -0.39 & 1.22 & 124.2 & -19.2 & -81.1 & -123.4 & -24.1 & 12.67 & 0.42 & 7.97 & 1.01 & 0.94 \\
\hline SS Cnc & -9.89 & -0.48 & 0.73 & 43.7 & 216.2 & -0.8 & -54.1 & 213.8 & 11.83 & 8.26 & 0.83 & 0.07 & 0.18 \\
\hline TT Cnc & -9.48 & -0.61 & 0.62 & -117.3 & 82.5 & -238.5 & 111.7 & 89.8 & 17.15 & 7.52 & 16.00 & 2.25 & 0.39 \\
\hline AQ Cnc & -9.65 & -0.90 & 1.15 & -256.7 & -217.0 & 16.9 & 275.8 & -192.2 & 34.69 & 4.35 & 3.68 & 0.11 & 0.78 \\
\hline W CVn & -8.40 & 0.30 & 0.90 & 1.1 & 142.2 & 83.8 & 3.9 & 142.2 & 8.45 & 4.65 & 2.47 & 0.30 & 0.29 \\
\hline $\mathrm{ZCVn}$ & -8.81 & 0.45 & 1.83 & 118.0 & -65.9 & 115.3 & -121.2 & -59.7 & 11.02 & 2.27 & 8.43 & 1.23 & 0.66 \\
\hline RZ CVn & -8.33 & 0.31 & 1.56 & -297.2 & -19.1 & 80.0 & 296.3 & -30.2 & 24.56 & 0.50 & 11.10 & 0.51 & 0.96 \\
\hline SS CVn & -8.44 & 0.52 & 1.66 & 281.0 & 0.1 & 30.1 & -280.5 & 17.3 & 21.39 & 0.24 & 12.44 & 0.76 & 0.98 \\
\hline ST CVn & -8.27 & 0.25 & 1.30 & -18.3 & 85.0 & -91.2 & 20.9 & 84.4 & 8.38 & 2.41 & 3.96 & 0.54 & 0.55 \\
\hline UZ CVn & -8.88 & 0.32 & 1.98 & 102.4 & -149.8 & 59.3 & -107.8 & -146.0 & 11.06 & 4.72 & 4.04 & 0.39 & 0.40 \\
\hline AA CMi & -9.50 & -0.68 & 0.14 & -42.1 & 216.6 & 25.1 & 26.4 & 219.1 & 10.45 & 8.77 & 0.43 & 0.04 & 0.09 \\
\hline AL CMi & -9.87 & -0.94 & 0.46 & -74.0 & 252.4 & -34.6 & 49.7 & 258.3 & 15.89 & 9.43 & 1.28 & 0.08 & 0.26 \\
\hline RV Cap & -7.71 & 0.51 & -0.67 & 56.8 & -325.6 & -195.6 & -78.3 & -321.1 & 38.47 & 7.56 & 17.69 & 0.52 & 0.67 \\
\hline YZ Cap & -7.74 & 0.54 & -0.77 & 49.6 & 95.7 & 114.4 & -42.9 & 98.9 & 8.21 & 2.77 & 3.77 & 0.52 & 0.50 \\
\hline IU Car & -8.51 & -1.47 & -0.62 & -236.3 & -77.5 & -91.2 & 246.1 & -36.1 & 17.68 & 0.75 & 7.80 & 0.49 & 0.92 \\
\hline BI Cen & -8.03 & -1.03 & 0.05 & 47.7 & 21.4 & 17.1 & -50.0 & 15.2 & 8.33 & 0.20 & 5.53 & 1.49 & 0.95 \\
\hline V499 Cen & -7.73 & -0.77 & 0.35 & 274.0 & -6.4 & -19.9 & -272.0 & -33.4 & 17.43 & 0.44 & 9.09 & 0.67 & 0.95 \\
\hline V671 Cen & -7.17 & -1.40 & 0.88 & 27.0 & -17.0 & -158.9 & -23.2 & -21.9 & 7.56 & 1.42 & 7.33 & 3.19 & 0.68 \\
\hline V674 Cen & -7.50 & -0.87 & 0.60 & -113.7 & -137.9 & 4.7 & 128.9 & -123.8 & 9.59 & 2.69 & 0.72 & 0.07 & 0.56 \\
\hline
\end{tabular}


G. Maintz and K. S. de Boer: RR Lyrae stars: kinematics, orbits and z-distribution, Online Material p 3

Table 1. continued.

\begin{tabular}{|c|c|c|c|c|c|c|c|c|c|c|c|c|c|}
\hline Name & $\begin{array}{r}X \\
{[\mathrm{kpc}]}\end{array}$ & $Y$ & $\bar{Z}$ & $\begin{array}{c}U \\
{\left[\mathrm{~km} \mathrm{~s}^{-1}\right.}\end{array}$ & $\bar{V}$ & $\overline{\bar{W}}$ & $\bar{\Phi}$ & $\bar{\Theta}$ & $\begin{array}{c}R_{\mathrm{a}} \\
{[\mathrm{kpc}]}\end{array}$ & $R_{\mathrm{p}}$ & $z_{\max }$ & $\begin{array}{r}n z e \\
-\end{array}$ & $e c c$ \\
\hline RZ Cep & -8.64 & 0.38 & 0.04 & -297.8 & 111.7 & 243.5 & 302.4 & 98.4 & 53.05 & 3.75 & 4220 & 1.41 & 0.87 \\
\hline & & .90 & & -203.7 & & & & & 7.63 & & & 10 & \\
\hline RR Cet & .76 & 0.19 & -0.56 & 79.0 & 3.1 & 12.5 & -76.9 & 4.9 & 9.48 & 2.30 & & .07 & .61 \\
\hline $\mathrm{U}$ Cet & 8.72 & 0.23 & 1.60 & -11.3 & -19.6 & -72.6 & 10.7 & -19.9 & 9.66 & 0.32 & 6.39 & 1.25 & 0.94 \\
\hline$V$ Cet & 3.99 & 0.02 & 1.02 & 7.9 & 49.5 & 109.1 & -7.7 & 49.5 & 9.11 & 1.50 & 5.82 & 0.85 & 0.72 \\
\hline $\mathrm{X}$ Cet & 3.56 & 0.29 & 1.36 & 339.8 & -53.0 & -10.8 & -341.4 & -41.4 & 33.84 & 0.60 & 14.99 & 0.50 & 0.97 \\
\hline Z Cet & & & & -97.0 & 115.8 & 74.9 & 97.4 & & 0.78 & 3.69 & & .42 & 0.49 \\
\hline $\mathrm{J}$ Cet & 8.36 & 46 & 1.82 & -256.6 & & 63.0 & 260.0 & 54.1 & .25 & 1.12 & & 70 & .89 \\
\hline XZ Cet & 8.69 & -0.01 & 0.55 & -93.4 & 162.8 & -131.8 & 93.2 & 162.9 & 1.70 & 5.54 & 05 & 0.47 & 0.36 \\
\hline $\mathrm{X} \mathrm{Col}$ & 9.48 & 2.03 & 0.96 & -234.3 & -65.3 & -17.0 & 242.7 & -14.9 & 8.76 & 0.28 & & 2.02 & 0.97 \\
\hline $\mathrm{Col}$ & .84 & 0.78 & -0.60 & -270.3 & -181.7 & -113.2 & 285.2 & -157.2 & 32.99 & 3.30 & & 0.28 & 0.82 \\
\hline Com & 60 & & & -30.4 & & -56.5 & 29.9 & & 9.02 & & & .59 & .73 \\
\hline Com & 8.57 & 0.03 & 1.66 & -229.5 & -40.1 & -28.8 & 229.6 & -39.3 & 16.25 & 0.64 & & 0.67 & 0.92 \\
\hline Y Com & 8.32 & & 2.19 & -58.8 & 132.2 & -20.1 & & & 8.96 & 3.8 & 43 & .28 & 0.40 \\
\hline Com & 3.29 & -0.04 & 1.37 & 1.7 & -35.9 & -68.3 & -1.5 & -35.9 & 9.21 & 0.58 & 96 & 0.83 & .88 \\
\hline $\mathrm{V}$ CrA & & & & 34.1 & & 8 & 7.6 & 73.2 & 10 & 1.46 & & & .66 \\
\hline 4138 & 7.67 & 0.03 & 23 & -56.1 & 84.4 & 120.5 & 55.8 & 84.6 & .06 & 2.26 & & .59 & .56 \\
\hline V592 Cr & 6.66 & -0.34 & -0.49 & -285.9 & -107.5 & 34.2 & 291.1 & -92.7 & 18.05 & 1.31 & 14 & 0.12 & 0.87 \\
\hline $\mathrm{V} \mathrm{CrB}$ & 7.84 & & 0.99 & 55.2 & -27.1 & 0.6 & -5 & -21.7 & 8.42 & 0.30 & 29 & 1.20 & 0.93 \\
\hline $\mathrm{CrB}$ & .73 & & & -29.0 & & 88.3 & & & 08 & 5. & & & 0.23 \\
\hline Crt & 3.40 & & & -82.0 & 1.7 & -32.0 & 68.3 & 0.6 & 9.07 & 3.20 & & 14 & .48 \\
\hline X Crt & 8.35 & 0.98 & 1.1 & 153.8 & -14.0 & -115.9 & 151.1 & -31.9 & 12.55 & 0.52 & & 0.77 & 0.92 \\
\hline JY Cyg & 8.26 & & o & 56.7 & 221.8 & -11.3 & & & 9.61 & 7.70 & & .02 & 0.11 \\
\hline $\mathrm{ZCyg}$ & .48 & & & -25.6 & 9.4 & -26 & & & & 8.33 & & & 0.36 \\
\hline & & & & 0.4 & & -26 & & & & 8. & & & \\
\hline & -7.50 & & 46 & 52.4 & & 16.4 & -34.7 & & 7.77 & 2.25 & 52 & 0.07 & 0.55 \\
\hline & -8.22 & 46 & & -54.8 & 209.7 & 6.6 & & & 9.84 & 6.31 & & 0.02 & 0.22 \\
\hline Dra & .44 & & & 21.1 & & 10.2 & -8.4 & & 8.54 & 2.12 & & 13 & 0.60 \\
\hline & .82 & & & -7.5 & & -3 & 3.2 & & 8.8 & 2. & & 06 & 50 \\
\hline & & & & -61.7 & & -2 & & & 64 & 3.90 & & .07 & .42 \\
\hline & & & 0.2 & -16.1 & & 5.0 & & & 9.51 & 7.71 & & 0.07 & 0.10 \\
\hline & & & & & & & & & & 0.7 & & 0.76 & \\
\hline & & & & -11 & & & & & & & & 57 & 0.72 \\
\hline & & & & 237.0 & & 6 & 30.6 & & 63 & 1. & & & \\
\hline & 8.91 & -0.28 & 0.33 & -4.9 & 10.3 & -73.2 & -1.7 & 10.4 & 8.94 & 8.91 & 57 & 0.19 & 0.00 \\
\hline & & & & & & & & & & 2.16 & & 0.07 & 0.64 \\
\hline & & & & & & & & & & 0.25 & & 1.09 & \\
\hline & & & & -12 & & & & 2.6 & & 6.39 & & 1.03 & 0.49 \\
\hline & .19 & & & & & & & 82.2 & & 1.99 & & 0.17 & 0.71 \\
\hline & & & & & & & & & & 0.25 & & 3.24 & 0.96 \\
\hline & & & & -42.7 & & & & -79.2 & 9.42 & 2.07 & & 0.23 & 0.64 \\
\hline & & & & -11 & & -57.4 & & 44.6 & 10.52 & 0. & & 0.72 & 0.84 \\
\hline & & & & -52.5 & & & & & 12.70 & 8.81 & & 0.06 & \\
\hline & -9.88 & & & -264.8 & & -14.8 & & & 22.16 & 0.60 & 80 & 0.52 & 0.95 \\
\hline & -7.07 & -0.12 & -1.58 & 84.8 & & 31.2 & -87.5 & 158.6 & 8.59 & 3.96 & 1.84 & 0.22 & 0.37 \\
\hline & & & & 1.1 & & -7 & & & .15 & 7.74 & 61 & 0.06 & 0.13 \\
\hline & & & & -360.8 & -40.0 & -2 & & -60.3 & 35.08 & 0.82 & & 0.08 & 0.95 \\
\hline & & & & & & & & 42.1 & 8.37 & 0.70 & & 0.77 & 0.84 \\
\hline & & & & -282.4 & -200.8 & & & -228.2 & 31.23 & 4.59 & 94 & 0.13 & 0.74 \\
\hline & -7.49 & & & -131.4 & -1 & & & -45.0 & 9.20 & 1.11 & .30 & 0.99 & 0.78 \\
\hline & & & & -11 & & & & 92.8 & 10.11 & 2.09 & & 0.48 & 0.66 \\
\hline & & & & & & & & & 9.42 & 3.12 & & 0.38 & 0.50 \\
\hline SZ Hya & -9.09 & -1.02 & 0.57 & 224.3 & -114.7 & -71.3 & -210.1 & -139.0 & 18.01 & 3.30 & 2.87 & 0.16 & 0.69 \\
\hline
\end{tabular}


G. Maintz and K. S. de Boer: RR Lyrae stars: kinematics, orbits and z-distribution, Online Material p 4

Table 1. continued.

\begin{tabular}{|c|c|c|c|c|c|c|c|c|c|c|c|c|c|}
\hline Name & $\begin{array}{c}X \\
{[\mathrm{kpc}]}\end{array}$ & $\bar{Y}$ & $Z$ & $\begin{array}{c}U \\
{\left[\mathrm{~km} \mathrm{~s}^{-1}\right.}\end{array}$ & $\bar{V}$ & $\bar{W}$ & $\Phi$ & $\Theta$ & $\left.\begin{array}{c}R_{\mathrm{a}} \\
{[\mathrm{kpc}]}\end{array}\right]$ & $R_{\mathrm{p}}$ & $z_{\max }$ & $\begin{array}{r}n z e \\
- \\
\end{array}$ & $\begin{array}{r}e c c \\
- \\
\end{array}$ \\
\hline WZ Hya & -8.73 & -0.81 & 0.58 & -52.8 & -55.4 & 113.0 & 57.7 & -50.3 & 9.32 & 1.34 & 5.50 & 0.76 & 0.75 \\
\hline D Hya & .91 & 1.18 & 0.64 & -67.7 & 90.4 & 45.1 & 56.6 & 97.8 & 10.46 & 2.81 & 34 & 13 & 0.58 \\
\hline H Hya & 9.44 & -1.51 & 0.75 & -268.6 & -48.4 & 2.3 & 272.9 & -5.4 & 21.92 & 0.12 & 17.45 & 1.59 & 0.99 \\
\hline IK Hya & 8.29 & -0.55 & 0.40 & -29.3 & -136.6 & -26.0 & 38.3 & -134.3 & 8.52 & 3.67 & 0.61 & 0.07 & 0.40 \\
\hline ET Hya & 9.49 & -1.34 & 0.55 & -140.3 & -83.5 & -17.1 & 150.6 & -63.0 & 12.41 & 1.38 & 1.47 & 0.14 & 0.80 \\
\hline K Hya & 3.29 & -0.55 & 0.40 & -29.1 & -136.4 & -26.0 & 38.3 & -134.2 & 8.52 & 3.67 & 0.61 & 0.07 & 0.40 \\
\hline V Ind & 7.99 & -0.04 & -0.48 & 306.6 & -55.6 & 53.4 & -306.3 & -57.2 & 23.85 & 0.84 & 2.89 & 0.14 & 0.93 \\
\hline RR Leo & 9.04 & -0.29 & 0.81 & -88.5 & 154.4 & 24.2 & 83.5 & 157.2 & 10.46 & 4.71 & 1.04 & 0.10 & 0.38 \\
\hline RX Leo & -9.01 & -0.29 & 1.66 & 242.7 & 57.2 & -79.6 & -244.4 & 49.4 & 19.38 & 1.08 & 8.43 & 0.49 & 0.89 \\
\hline Leo & 8.55 & -0.67 & 1.03 & -51.6 & -17.3 & 36.0 & 52.8 & -13.3 & 9.21 & 0.21 & 6.30 & 1.48 & 0.95 \\
\hline Leo & .66 & -0.53 & 1.26 & 43.8 & -66.6 & 68.1 & -39.6 & -69.2 & 9.05 & 1.71 & 4.02 & 0.54 & 0.68 \\
\hline A Leo & .73 & -0.80 & 1.87 & 55.0 & -79.2 & -85.5 & -47.6 & -83.9 & 9.80 & 2.19 & .01 & 0.47 & 0.64 \\
\hline AE Leo & -9.03 & -0.73 & 2.24 & 324.5 & 52.0 & -34.9 & -327.7 & 25.7 & 33.48 & 0.43 & 26.03 & 1.31 & 0.97 \\
\hline AN Leo & 8.79 & -0.98 & 1.82 & 188.9 & -23.8 & -180.2 & -185.1 & -44.5 & 18.19 & 1.56 & 15.09 & 1.48 & 0.84 \\
\hline X Leo & 3.81 & -0.79 & 1.93 & -124.5 & -166.8 & 33.1 & 138.8 & -155.1 & 13.01 & 4.22 & 2.61 & 0.20 & 0.51 \\
\hline K Leo & .75 & -0.45 & 1.41 & -95.3 & 62.8 & -37.8 & 91.9 & 67.6 & 9.75 & 1.57 & .87 & 0.21 & 0.72 \\
\hline V LMi & -9.27 & -0.30 & 1.32 & 321.8 & 41.9 & 16.6 & -323.0 & 31.5 & 31.42 & 0.47 & 17.15 & 0.68 & 0.97 \\
\hline X LMi & 9.80 & -0.06 & 1.77 & 150.2 & 37.7 & 2.8 & -150.4 & 36.9 & 13.21 & 0.69 & 7.02 & 0.73 & 0.90 \\
\hline U Lep & 9.11 & -0.53 & -0.55 & 113.0 & -114.3 & 8.9 & -106.2 & -120.7 & 10.74 & 3.18 & 0.63 & 0.06 & 0.54 \\
\hline$Y \mathrm{Lib}$ & .31 & -0.13 & 0.66 & 227.0 & -15.4 & -119.4 & -226.7 & -19.3 & 14.94 & 0.23 & 10.17 & .13 & 0.97 \\
\hline G Lib & 7.40 & -0.31 & 0.54 & -297.4 & -72.7 & 38.7 & 300.2 & -60.1 & 20.76 & 0.84 & 9.41 & 0.54 & 0.92 \\
\hline FW Lup & -8.18 & -0.18 & 0.09 & 4.0 & 209.3 & -8.6 & -8.6 & 209.2 & 8.24 & 7.38 & 0.13 & 0.02 & 0.06 \\
\hline TT Lyn & -9.02 & 0.04 & 0.47 & -131.1 & 100.9 & -237.7 & 131.5 & 100.3 & 18.14 & 6.59 & 16.43 & 1.94 & 0.47 \\
\hline$N$ Lyn & 9.96 & 0.10 & 0.76 & 23.3 & 254.2 & -53.4 & -20.8 & 254.4 & 15.15 & 9.82 & 2.13 & 0.14 & 0.21 \\
\hline Lyr & .44 & 0.24 & 0 . & 254.5 & 102.2 & 9.3 & -251.4 & 109.4 & 18.36 & 2.08 & .21 & 0.01 & 0.80 \\
\hline RZ Lyr & -7.85 & 1.23 & 0.39 & -271.9 & 146.1 & -104.0 & 291.2 & 102.3 & 24.57 & 1.90 & 8.44 & 0.36 & 0.86 \\
\hline N Lyr & -7.97 & 0.85 & 0.26 & 59.9 & 240.3 & -3.5 & -34.2 & 245.3 & 10.64 & 7.70 & 0.32 & 0.03 & 0.16 \\
\hline EZ Lyr & 7.95 & 1.21 & 0.39 & -92.8 & 190.8 & 27.8 & 120.3 & 174.7 & 10.86 & 4.47 & 0.65 & 0.06 & 0.42 \\
\hline Lyr & 7.78 & 1.28 & 54 & -257.0 & 112.7 & 111.6 & 272.0 & 69.2 & 20.04 & 1.64 & 8.87 & 0.35 & 0.85 \\
\hline KX Lyr & 8.16 & 0.86 & 0.34 & -73.4 & 212.0 & 3.9 & 95.2 & 203.2 & 10.86 & 5.75 & 0.41 & 0.04 & 0.31 \\
\hline Z Mic & -7.54 & 0.27 & -0.94 & -100.2 & 173.8 & -33.5 & 106.3 & 170.1 & 9.76 & 4.28 & 1.25 & 0.13 & 0.39 \\
\hline RV Oct & -7.99 & -0.75 & -0.36 & -8.4 & 23.4 & 52.9 & 6.2 & 24.1 & 8.24 & 0.36 & .25 & 1.28 & 0.92 \\
\hline Y Oct & 7.50 & -1.01 & 1.00 & -32.8 & 19.3 & 19.9 & 29.9 & 23.5 & 7.74 & 0.75 & 6.51 & 1.89 & 0.82 \\
\hline & 7.62 & -1.09 & 0.88 & 57.2 & -175.8 & -22.5 & -31.8 & -182.1 & 7.98 & 5.59 & 02 & 0.13 & 0.18 \\
\hline UV Oct & -8.19 & -0.40 & -0.22 & -213.4 & -34.3 & -116.5 & 214.8 & -23.9 & 15.50 & 0.33 & 9.99 & 1.00 & 0.96 \\
\hline UW Oct & -7.39 & -1.27 & -1.11 & 105.7 & 189.4 & 35.8 & -136.3 & 168.7 & 11.00 & 3.96 & .58 & 0.14 & 0.47 \\
\hline ST Oph & -7.20 & & & 18.3 & 238.8 & 19.2 & -0.1 & 239.5 & 8.79 & 7.22 & 0.56 & 0.06 & 0.10 \\
\hline V445 Oph & -7.78 & .10 & 0.39 & -29.2 & 220.7 & 41.2 & 32.0 & 220.3 & 8.89 & 7.14 & 0.81 & 0.09 & 0.11 \\
\hline V455 Oph & -7.19 & 1.10 & 0.41 & 146.3 & -33.1 & 154.4 & -149.6 & -10.5 & 10.16 & 0.57 & 9.71 & 4.76 & 0.89 \\
\hline TY Pav & -6.51 & -1.12 & -0.70 & -6.0 & -290.1 & -31.4 & 55.1 & -284.8 & 13.27 & 6.36 & 1.34 & 0.10 & 0.35 \\
\hline WY Pav & -7.00 & -0.66 & -0.46 & -152.8 & -33.8 & 148.8 & 155.3 & -19.3 & 9.88 & 0.43 & 8.99 & 2.55 & 0.92 \\
\hline & .82 & -0.98 & .82 & 395.5 & 121.4 & -0.9 & -408.8 & 63.8 & 49.41 & 0.76 & 18.88 & 0.41 & 0.97 \\
\hline & -6.74 & -0.78 & 1.07 & 77.2 & 155.1 & -58.2 & -94.4 & 145.3 & 7.99 & 3.49 & 1.95 & 0.25 & 0.39 \\
\hline BP Pav & -6.83 & -0.95 & -1.17 & 241.3 & -259.1 & 69.8 & -203.5 & -289.8 & 28.78 & 5.14 & 4.93 & 0.17 & 0.70 \\
\hline VV Peg & -8.21 & 1.44 & -0.86 & -37.1 & 204.9 & -84.0 & 72.0 & 195.4 & 10.65 & 6.19 & 2.55 & 0.24 & 0.26 \\
\hline VZ Peg & -8.85 & 1.4 & .04 & 26.3 & -150.2 & -68.8 & -49.9 & -144.1 & 9.39 & 4.79 & 2.48 & 0.27 & 0.32 \\
\hline AV Peg & -8.35 & 0.67 & -0.31 & -21.9 & 165.8 & -16.5 & 35.1 & 163.6 & 8.62 & 4.91 & 0.39 & 0.04 & 0.27 \\
\hline BH Peg & -8.45 & 0.64 & -0.51 & 204.5 & -94.2 & 55.2 & -211.0 & -78.5 & 14.53 & 1.51 & 1.63 & 0.11 & 0.81 \\
\hline CG Peg & -8.28 & 0.97 & -0.38 & 33.6 & 218.9 & -2.6 & -7.8 & 221.4 & 8.66 & 8.21 & 0.39 & 0.04 & 0.03 \\
\hline DH Peg & -8.35 & 0.39 & -0.34 & -46.5 & 172.8 & 22.9 & 54.5 & 170.5 & 8.98 & 5.11 & 0.52 & 0.06 & 0.27 \\
\hline DZ Peg & -8 & 1.3 & -1.20 & 46.0 & -115.2 & 53.9 & -63.4 & -106.7 & 9.32 & 2.84 & 1.88 & 0.20 & 0.53 \\
\hline AR Per & -9.05 & 0.26 & -0.02 & 4.7 & 232.3 & -26.4 & 1.9 & 232.4 & 10.26 & 9.05 & 0.40 & 0.04 & 0.06 \\
\hline RV Phe & -7.75 & -0.34 & -1.69 & -307.7 & -63.4 & -184.6 & 310.2 & -50.0 & 36.63 & 0.82 & 19.65 & 0.64 & 0.96 \\
\hline
\end{tabular}


G. Maintz and K. S. de Boer: RR Lyrae stars: kinematics, orbits and z-distribution, Online Material p 5

Table 1. continued.

\begin{tabular}{|c|c|c|c|c|c|c|c|c|c|c|c|c|c|}
\hline Name & $\begin{array}{r}X \\
{[\mathrm{kpc}]}\end{array}$ & $Y$ & $\bar{Z}$ & $\begin{array}{c}U \\
{\left[\mathrm{~km} \mathrm{~s}^{-1}\right]}\end{array}$ & 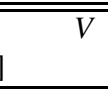 & $\overline{\bar{W}}$ & $\Phi$ & $\bar{\Theta}$ & $\begin{array}{c}R_{\mathrm{a}} \\
{[\mathrm{kpc}]}\end{array}$ & $R_{\mathrm{p}}$ & $z_{\max }$ & $\begin{array}{r}n z e \\
- \\
\end{array}$ & $\begin{array}{r}e c c \\
- \\
\end{array}$ \\
\hline J Pic & -8.72 & -0.99 & -0.84 & -0.7 & 229.2 & -15.3 & -25.1 & 227.8 & 10.23 & 8.46 & 1.01 & 0.10 & 0.09 \\
\hline & & & & 174.3 & & & & & & & & & \\
\hline & .67 & & & 281.3 & -8.4 & -85.7 & 78.8 & -38.0 & 60 & 0.67 & & 55 & 94 \\
\hline Psc & .07 & 0.63 & 0.73 & 11.4 & 228.2 & -6.7 & 4.5 & 228.5 & 0.13 & 9.09 & 0.80 & .08 & 0.05 \\
\hline XX Pup & .16 & -1.00 & 0.18 & 251.0 & -74.0 & -31.3 & 257.5 & -46.2 & 9.36 & 0.80 & 9.29 & .58 & 0.92 \\
\hline H Pup & 3.70 & 0.95 & -0.26 & 13.9 & 223.0 & 14.3 & -38.1 & 220.2 & 0.01 & 7.80 & 0.35 & .04 & 0.12 \\
\hline & 16 & & 0.10 & -85.1 & & 120.4 & & 46.0 & & & & 86 & .77 \\
\hline 38 Pup & .64 & 0.48 & -0.20 & 79.2 & -119.0 & 27.7 & -72.5 & 123.2 & 9.36 & 3.23 & 46 & 05 & .49 \\
\hline X Ret & 3.29 & 1.07 & 1.09 & -291.4 & -35.0 & 10.9 & 293.5 & 2.7 & 22.07 & 0.11 & 17.93 & 1.69 & 0.99 \\
\hline $440 \mathrm{Sgr}$ & .79 & 0.20 & 0.26 & -12.8 & 26.1 & -25.0 & 13.5 & 25.8 & 7.83 & 0.38 & 4.87 & 1.26 & 0.91 \\
\hline 675 & .71 & -0.02 & & -113.8 & 220.1 & -117.6 & & 220.4 & 13.58 & 5.84 & & 30 & 0.40 \\
\hline 96 & 5.81 & 0.03 & & .7 & -66.4 & & & -65.5 & & & 32 & 37 & .83 \\
\hline 1646 & 7.04 & 0.29 & 0.94 & 206.5 & -149.0 & -73.1 & -212.6 & -140.2 & 13.59 & 2.81 & 4.55 & .35 & 0.66 \\
\hline V494 S & 7.84 & -0.03 & & 34.6 & & -18.7 & & & & 6.73 & 21 & 03 & 0.12 \\
\hline 19030 & & -0.14 & & -56.6 & 197.1 & 33.8 & 52.9 & & & 5.81 & & & .19 \\
\hline & 38 & & & 3.3 & & -67.2 & & & & 1.92 & 02 & 20 & 70 \\
\hline & .70 & -0.23 & & 78.6 & 57.5 & 24.2 & & 52.6 & 3.08 & 0.96 & 45 & & 86 \\
\hline $\mathrm{Scl}$ & 7.83 & 0.23 & 198 & 55.6 & 7.7 & 1.3 & -55.4 & 9.3 & 8.27 & 0.23 & 84 & 32 & 0.94 \\
\hline N Scl & .46 & -0.25 & & 23.3 & -13.6 & 12.0 & & -14.3 & 8. & 0.20 & 95 & 49 & .96 \\
\hline & 61 & .42 & & 118.8 & & & & & & & 12 & & .36 \\
\hline & .50 & .26 & & 6.0 & & 10.5 & & & 2.95 & 0.82 & & 67 & 93 \\
\hline $\mathrm{Ser}$ & 7.94 & 06 & 0.55 & -261.5 & -61.2 & 109.5 & 61.0 & & 8.58 & 1.49 & 76 & 38 & .85 \\
\hline & & & & -7.3 & & & & & 7.98 & 6.47 & 84 & & .10 \\
\hline A & 78 & & 0.96 & -13.3 & -10 & -2 & & & & & 12 & & 52 \\
\hline & & & & & & & & & & & & & \\
\hline & .44 & & & 6 & & .0 & & & & 5.5 & & 20 & 0.16 \\
\hline & .56 & & 0.71 & -27.5 & & & & & 8.82 & 7.08 & 6 & 10 & 0.11 \\
\hline & 80 & 0.45 & & -22.2 & & -5 & & & 8.86 & 4.80 & & 13 & 0.30 \\
\hline & 80 & 4 & & -108.9 & & & & & 0.62 & 4. & & 20 & 40 \\
\hline & & & & & & & & & & 2.52 & & & 52 \\
\hline & & & & -39.0 & & 130.3 & & & 11 & 6.10 & & 49 & .14 \\
\hline & & & & 79.1 & & & & & & 4.96 & & & 0.34 \\
\hline & & & & & & & & & & 6.9 & & & \\
\hline & & & & & & & & & & 2. & & & \\
\hline$T_{u}$ & .57 & 64 & 56 & & & & -59.1 & 9 & 8.74 & 0.05 & 51 & 35 & 99 \\
\hline & & & & & & & & & & & & & 0.68 \\
\hline & & & & & & & & & & & & & \\
\hline & & -1.59 & & 218.6 & & & & & 16.82 & 1. & & 42 & 0.79 \\
\hline & 66 & & & & & & 9 & & & 1.30 & 67 & .21 & 0.75 \\
\hline & & & & & & & & & & & & & 0.89 \\
\hline & & & & & & & & & & 0.1 & & 1.40 & 0.97 \\
\hline & & & & & & & & & & 3. & 6 & & 0.46 \\
\hline & & & & & & & & & & 0.46 & & 69 & 0.96 \\
\hline & 8.23 & & & & & & & & 1.00 & 0.57 & & 1.14 & 0.97 \\
\hline & 7.73 & & & & & & & & 26 & 3.20 & 1.39 & .17 & 0.44 \\
\hline & 8.42 & & & & & & & & & 3.66 & 1.71 & 0.15 & 0.52 \\
\hline & & & & & & & & & & 1.46 & 64 & & 0.70 \\
\hline & & & & .5 & & & & -65.6 & & 0.98 & & 0.43 & 0.94 \\
\hline & & & & & & & & 0.4 & & 0.01 & & 2.69 & 1.00 \\
\hline & 702 & & & & & & & -36.2 & 36 & 0.51 & 14 & 0.62 & 0.95 \\
\hline & & & & & -254 & & & -240.5 & 36.76 & 5.99 & & 0.63 & 0.72 \\
\hline & & & & & & 3.6 & & 21.0 & & 0.29 & & 1.28 & 0.94 \\
\hline & -8.06 & & & & & 50.9 & -272.7 & 2.2 & 19.64 & 0.06 & 17.91 & 6.07 & 0.99 \\
\hline & -8.01 & & & -207.9 & & & & 49.9 & 13.40 & 0.79 & 6.15 & 0.59 & 0.89 \\
\hline & & & & & & & & & 32 & 2.66 & 2.84 & 0.23 & 0.64 \\
\hline & & -1.01 & & & & & & & 10.24 & 2.88 & 4.91 & 0.55 & 0.56 \\
\hline & -0.12 & 0.63 & & & -91.0 & & & & 8.38 & 2.05 & 0.95 & 0.13 & 0.61 \\
\hline FK Vul & -7.64 & 2.09 & -0.56 & 204.5 & 55.7 & -45.7 & -182.6 & 107.7 & 12.16 & 2.23 & 1.67 & 0.14 & 0.69 \\
\hline
\end{tabular}

